\title{
Knockdown of the Candidate Dyslexia Susceptibility Gene Homolog Dyx1c1 in Rodents: Effects on Auditory Processing, Visual Attention, and Cortical and Thalamic Anatomy
}

\author{
Caitlin E. Szalkowski ${ }^{a} \quad$ Anne B. Booker ${ }^{b}$ Dongnhu T. Truong ${ }^{a} \quad$ Steven W. Threlkeld ${ }^{c}$ \\ Glenn D. Rosen ${ }^{d}$ Roslyn H. Fitch ${ }^{a}$
}

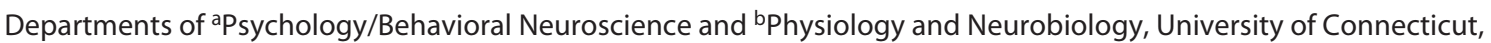
Storrs, Conn., 'Department of Psychology, Rhode Island College, Providence, R.I., and 'Department of Neurology, Beth Israel Deaconess Medical Center, Boston, Mass., USA

\section{Key Words}

RNA interference · Medical geniculate nucleus · Rapid auditory processing $\cdot$ Attention

\begin{abstract}
The current study investigated the behavioral and neuroanatomical effects of embryonic knockdown of the candidate dyslexia susceptibility gene (CDSG) homolog Dyx1c1 through RNA interference (RNAi) in rats. Specifically, we examined long-term effects on visual attention abilities in male rats, in addition to assessing rapid and complex auditory processing abilities in male and, for the first time, female rats. Our results replicated prior evidence of complex acoustic processing deficits in Dyx $1 c 1$ male rats and revealed new evidence of comparable deficits in Dyx1c1 female rats. Moreover, we found new evidence that knocking down Dyx1c1 produced orthogonal impairments in visual attention in the male subgroup. Stereological analyses of male brains from prior RNAi studies revealed that, despite consistent visible evidence of disruptions of neuronal migration (i.e., heterotopia), knockdown of $D y x 1 c 1$ did not significantly alter the cortical volume, hippocampal volume, or midsagittal area of the corpus callosum (measured in a separate cohort of like-treat-
\end{abstract}

ed Dyx1c1 male rats). Dyx1c1 transfection did, however, lead to significant changes in medial geniculate nucleus (MGN) anatomy, with a significant shift to smaller MGN neurons in Dyx1c1-transfected animals. Combined results provide important information about the impact of $D y \times 1 c 1$ on behavioral functions that parallel domains known to be affected in language-impaired populations as well as information about widespread changes to the brain following early disruption of this CDSG.

Copyright $\odot 2013$ S. Karger AG, Basel

\section{Introduction}

Developmental dyslexia is a common disorder affecting roughly $5-10 \%$ of the population worldwide [1] and is characterized by a significant impairment in reading despite adequate intelligence and educational opportunity. Deficits in more basic 'core' component behaviors - also termed 'intermediate phenotypes' - are also common in dyslexic individuals, including deficits in phonological processing [2-4], short-term and/or working memory [5-9], visuospatial attention [10, 11], and rapid auditory processing [12-15]. The underlying

\section{KARGER}

E-Mail karger@karger.com

www.karger.com/dne
(C) 2013 S. Karger AG, Basel

0378-5866/13/0351-0050\$38.00/0
Caitlin E. Szalkowski

University of Connecticut

406 Babbidge Road, Unit 1020

Storrs, CT 06269 (USA)

E-Mail caitlin.szalkowski@gmail.com 
cause(s) of these behavioral impairments and the associated dyslexia diagnosis remains unknown. However, a study by Galaburda et al. [16] revealed microscopic changes in perisylvian regions of the cortex in the postmortem brains of 4 dyslexic males, providing the first evidence to suggest that the neurological foundation for dyslexia may become evident early in brain development and specifically during the process of neuronal migration. In the same brains, Galaburda et al. [17] also noted a significant shift in the distribution of large and small cells in the medial geniculate nucleus (MGN) of the thalamus of dyslexic individuals (as compared to brains of unaffected subjects) - a finding that had significant functional implications given the known role of the MGN in auditory processing, coupled with the prevalence of auditory processing deficits in dyslexia [17]. Similar examples of cortical migrational disruption were observed postmortem in the brains from 3 female dyslexics, although females had fewer ectopias [18]. More recent studies have used neuroimaging to examine the brains of dyslexic individuals and have revealed a wide (and at times contradictory) array of structural/functional changes associated with the disorder, including region-specific reductions in the gray matter [19-23], changes in white matter organization [24], region-specific changes in the size of the corpus callosum [25-27], and changes in patterns of activation during language/reading tasks, all of which are suggestive of subtle but widespread changes in connectivity [28-33].

Genetic and epidemiological studies have identified several genes as candidate dyslexia susceptibility genes (CDSGs), specifically through genetic linkage and association studies of affected populations in several countries [34-41]. Building upon these findings, researchers have developed animal models of genetic disruption targeting the rodent homologs of a subset of these genes (Dyx1c1, Kiaa0319, and Dcdc2). One such model utilizes 'knockdown' rats created via in utero electroporation of hairpin RNA interference (RNAi) for the target gene(s). Data derived from these animal models revealed that each of these 3 genes examined plays a critical role in cortical development and, more precisely, in the process of neuronal migration [38,42-44]. This is a striking result given the neuronal migration anomalies observed in the brains of human dyslexics. However, effects of disruption of these genes on more specific gross morphometric measures in the brain (such as regional volumes and thalamic cell size distribution) have not yet been examined using animal models.

To date, few studies have explored the associations between specific intermediate phenotypes of dyslexia and
CDSG variants. Variants of one gene, DYX1C1, have been explicitly linked to memory deficits in some languageimpaired populations [45-48]. DYX1C1 variants have also recently been linked to deficits in mathematical mental calculation skills in dyslexic individuals [49]. In the brain, DYX1C1 variants have recently been reported to correlate with increases in the gray matter volume in the cerebellum in a sample of healthy and schizophrenic individuals [50]. Using the RNAi animal model, our lab has previously demonstrated that knocking down Dyx1c1 leads to deficits in rapid auditory processing and spatial working memory in male rats $[51,52]$. Impacts of this knockdown on other behavioral domains and on gross brain morphology have not yet been explored.

The current study sought to better characterize the role of Dyx1c1 in rodent brain development and behavior. Male rats transfected with Dyx1c1 shRNA and shamtreated counterparts were tested on an operant visual attention paradigm. Since we have already reported effects of Dyx1c1 knockdown on complex auditory processing in male rats [51], the task was replicated in the current study using both male and female rats. In previous studies, males were used exclusively for testing based on prior research that has demonstrated that males exhibit more robust behavioral deficits following disruptions of neuronal migration [53-55]. The inclusion of female rats has relevance to dyslexia, which has been shown to disproportionately affect males as compared to females (estimates range from 1.5:1 to 3:1 [1]). Male rats transfected with Dyx1c1 shRNA were expected to show deficits on tasks of rapid and complex auditory processing (replicating prior reports, see [51]); however, possible effects on females could not be predicted. Note that for visual attention testing, equipment limitations allowed us to test only a subset of animals, and we chose to assess males since we have in hand more data behaviorally characterizing Dyx1c1 shRNA-transfected males. Future studies will be needed to assess attention in Dyx1c1 shRNAtransfected females. To our knowledge, this was the first assessment of attention in a CDSG rodent model. Finally, in order to better understand the effects of this genetic disruption on widespread brain morphology, we performed stereological analyses on histological tissue samples from like-treated Dyx1c1 and sham male animals from two prior behavioral studies (in which males were used exclusively), specifically taking measurements of the cortical volume, hippocampal volume, midsagittal corpus callosum area, and neuronal cell size in the MGN $[51,52]$. 


\section{Experimental Procedures}

A total of 18 female and 17 male Wistar rats (Charles River Laboratory, Wilmington, Mass., USA) were used for behavioral testing (female sham, $\mathrm{n}=11$; female Dyx1c1 shRNA, $\mathrm{n}=7$; male sham, $\mathrm{n}=8$; male Dyx1c1 shRNA, $\mathrm{n}=9$ ), and treatments were within-litter to minimize litter effects. Rats were pair-housed in Plexiglas tubs with same-sex littermates until postnatal day 45 (P45), at which time they were single-housed. They were maintained on a 12-/12-hour light/dark cycle, and food and water were available ad libitum. All procedures were performed in accordance with the guidelines established by the National Institutes of Health and were approved by the University of Connecticut's Institutional Care and Use Committee.

\section{In utero Electroporation}

In utero electroporation of Dyx1c1 shRNA for the 18 female and 17 male rats was performed by one of the authors (A.B.B.) at the University of Connecticut in accordance with the procedures described in Bai et al. [56]. (For details on the transfection of the males used for stereological analyses, see [51, 52].) All surgeries were performed on embryonic day 15.5 (E15.5). In all Dyx1c1 shRNA treatments, plasmids encoding Dyx1c1 short hairpin RNA (pU6DyxHPB; $1.5 \mu \mathrm{g} / \mu \mathrm{l}$ ), $0.5 \mu \mathrm{g} / \mu \mathrm{l}$ piggyBac-encoded red fluorescent protein (RFP), and $1 \mu \mathrm{g} / \mu \mathrm{l}$ piggyBac transposase were cotransfected into the fetal ventricular zone. For sham treatments, animals received ventricular transfection of $1.5 \mu \mathrm{g} / \mu \mathrm{l}$ piggyBacencoded green fluorescent protein (GFP) and $1.5 \mu \mathrm{g} / \mu \mathrm{l}$ piggyBac transposase. The use of piggyBac vectors causes the associated fluorescent markers (RFP and GFP) to become incorporated into the transfected cell's DNA, and thus passed on to daughter cells (see [57]). This ensured a detectable GFP or RFP signal after sacrifice, which was important given that the animals survived for several months after the embryonic transfection surgery.

Pregnant dams were heavily anesthetized with $100 \mathrm{mg} / \mathrm{kg}$ ketamine and $15 \mathrm{mg} / \mathrm{kg}$ xylazine injected intraperitoneally. A longitudinal midline incision was made in the lower abdomen, and the uterine horns were exposed. Within each litter (fetuses could not be sexed), half of the pups received an injection of the experimental plasmid solution, while the other half received a control injection. Further, $1-3 \mu \mathrm{l}$ of plasmid mixed with Fast Green $(2 \mathrm{mg} / \mathrm{ml}$, Sigma) were microinjected via air pressure (General Valve Picospritzer, Pine Brook, N.J., USA) into one randomly chosen lateral ventricle of each embryo using a pulled glass pipette as a needle. Following injection, the plasmids were electroporated by discharging a $500-\mu \mathrm{F}$ capacitor charged at $70 \mathrm{~V}$ with a sequencing power supply across a pair of copper alloy plates, creating a favorable electrical environment for the plasmid solutions to be taken into the nuclei of neural progenitor cells at the ventricular zone surface. Bilateral electroporation was achieved by switching the position of the copper alloy plates around the head of the embryo and delivering a $70-\mathrm{mV}$ pulse to both the right and the left hemispheres. This resulted in some transfection of cells in each hemisphere, although the randomly chosen hemisphere that received the injection displayed the larger transfection in postmortem analysis. Paddles were positioned in such a way as to target cells in the ventricular zone that were destined for parietal cortex (for details, see [58]). When the entire litter was transfected, the uterine horns were returned to the abdominal cavity, and the muscle and skin were sutured closed. Each dam received injections of Metacam $(5 \mathrm{mg} / \mathrm{ml}$;
Butler Schein, Dublin, Ohio, USA) to alleviate postoperative pain, with recovery on a heating pad until return to the home cage where a palatable mash was provided.

The rats were weaned on P21, received right or left ear marking, and were housed in same-sex litter pairs (since treatment could not be identified at weaning). Treatment was later identified postmortem via observation of either RFP or GFP in histological preparations.

\section{Auditory Testing}

Startle Reduction Paradigm

It is important to note that experimenters were blind to the animals' treatment condition throughout testing. For a complete timeline of behavioral testing, refer to figure 1a. Auditory processing testing utilized a modified prepulse inhibition paradigm that has been discussed in detail elsewhere [59]. Briefly, in this paradigm a benign auditory cue is presented prior to a startle-eliciting stimulus (SES). The SES triggers an acoustic startle response (ASR), which is a gross motor reflex. If the rat is able to detect the pre-SES cue, the amplitude of the ASR is reduced. Thus, the rat's inhibition of the startle response acts as a mechanism to assess cue detectability [60]. During testing, the animals were placed on load cell platforms that recorded their movement (Med Associates, Georgia, Vt., USA). Acoustic stimuli were generated using a Pentium 4 Dell PC with custom sound programs and a Tucker Davis Technologies RP2 real-time processor. Sound files were played through a Marantz integrated amplifier that was connected to 9 Cambridge Soundworks speakers. The sound levels of each program used during testing were calibrated before testing (RadioShack Digital Sound Level Meter). Each pair of platforms had one speaker centered approximately 24 inches above it. The voltage output from each platform was passed through a load cell amplifier (PHM-250-60) into a Biopac MP100WS acquisition system (Biopac Systems, Santa Barbara, Calif., USA). The Biopac system was connected to two Macintosh Power Mac G4 computers running the program Acqknowledge v. 3.9.2. (Biopac Systems), which recorded the amplitude of each animal's ASR in millivolts following each presentation of the SES. Attenuated response scores (ATT scores) were calculated for each rat using the following formula: mean cued ASR/mean uncued ASR $\times 100$. This created a ratio for each animal of the amplitude of their displacement of the load cell platform on cued trials compared to the displacement amplitude on uncued trials. ATT scores were expressed as percentages and represented the main dependent variable of interest for auditory testing. In sessions where multiple cue types occurred in a session (e.g., gap detection), ATT scores were ascertained for each cue. When the cue was consistent within each test session [e.g., oddball, frequency-modulated (FM) sweep], only one ATT score per session was derived.

\section{Normal Single Tone}

Juvenile auditory testing began on P25 for all male and female animals. All rats were initially tested for 1 day on the normal single tone task, which provides a measure of individual differences in baseline hearing, gross motor startle response, and general prepulse inhibition. On uncued trials, this task consisted of a silent background that was interrupted by a $105-\mathrm{dB}, 50-\mathrm{ms}$ white noise SES. On cued trials, the SES was preceded by $50 \mathrm{~ms}$ by a single $75-$ $\mathrm{dB}, 7-\mathrm{ms}, 2,300-\mathrm{Hz}$ frequency tone pip. 


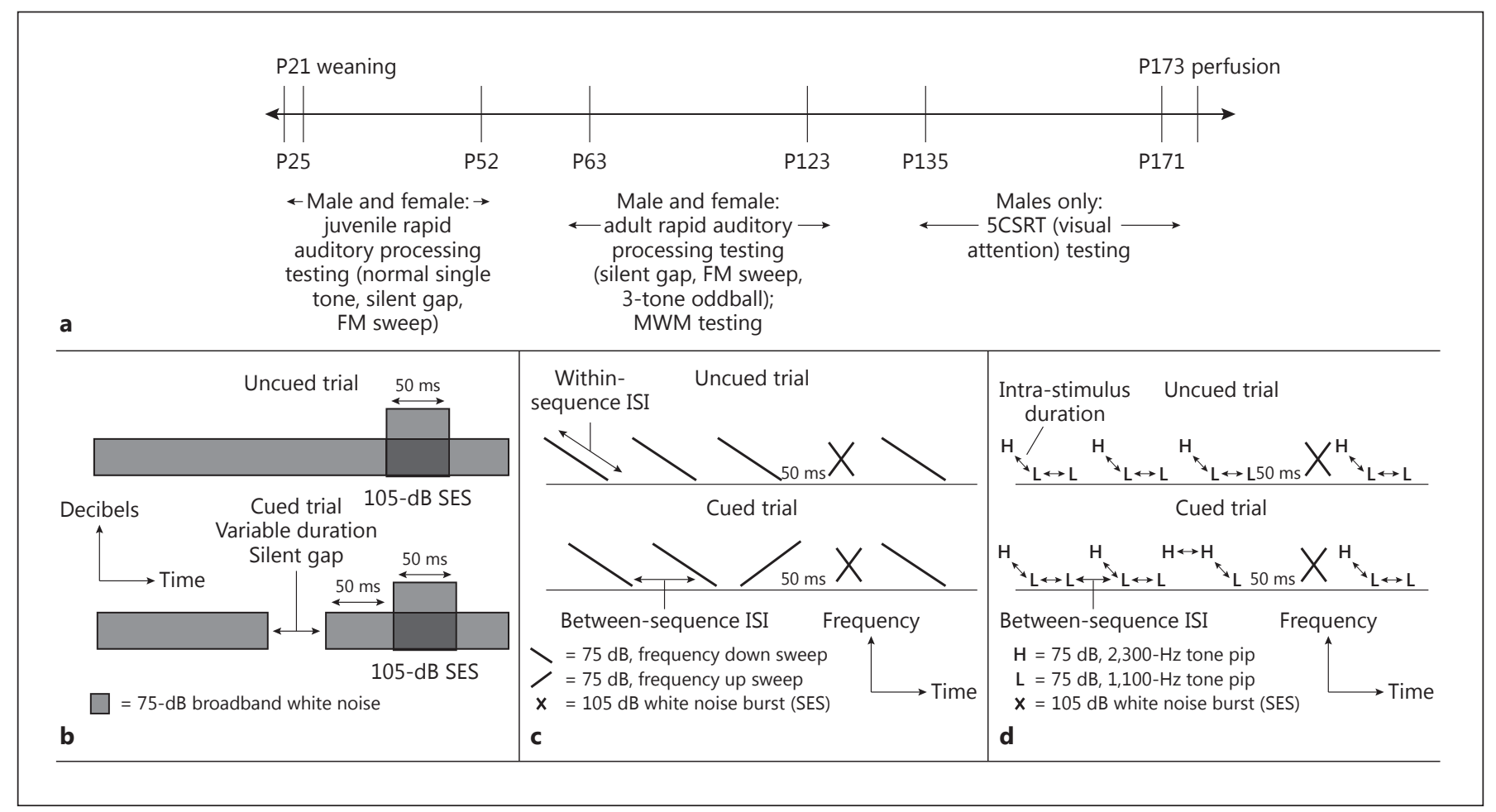

Fig. 1. Timeline of the behavioral testing of the male and female Wistar rats (a) and schematics of auditory processing tasks (b-d). b A schematic of the silent gap detection task. Silent gaps were of variable durations between 2 and $100 \mathrm{~ms}$. c A schematic of uncued and cued trials in the FM sweep task. In the cued trial, the cue is a reversal of the background frequency sweep from high-to-low to low-to-high, as depicted. The high frequency is $2,300 \mathrm{~Hz}$ and the low frequency is $1,100 \mathrm{~Hz}$. The intrastimulus duration progressed from long to short across days of testing, making the frequency sweep (and thus the cue) more difficult to discriminate. The between-sequence ISI was always $200 \mathrm{~ms}$ greater than the intrastimulus duration. $\mathbf{d}$ A schematic of the 3-tone oddball task. The intrastimulus duration and between-sequence ISI varied across weeks of testing, progressing from long to short to increase the temporal demands of the task.

\section{Silent Gap Detection}

A silent gap detection task was utilized during juvenile (P25$\mathrm{P} 45)$ and adult (P60+) testing over a period of 4 days. For a schematic of the silent gap task, refer to figure 1b. In this task, the background sound was a constant $75-\mathrm{db}$ broadband white noise. The cues that preceded the 105-db SES were silent gaps in the white noise background that ranged from 2 to $100 \mathrm{~ms}$ in duration. These gaps were presented in a pseudo-random order. On uncued trials, the broadband white noise background was not interrupted by a silent gap prior to SES presentation. The intertrial interval (ITI; the interval between the presentation of the SES and the start of the next trial) was between 16 and $24 \mathrm{~ms}$, and the length of each ITI varied in a pseudo-random order. One session of silent gap testing consisted of 300 trials.

\section{FM Sweep}

The FM sweep task was administered over 4 days, also in both the juvenile and adult periods. Each day of FM sweep testing consisted of 104 trials (cued and uncued) presented in a pseudo-random order. For a schematic of the FM sweep task, refer to figure $1 \mathrm{c}$. In this procedure, the background sound was a repeating $75-\mathrm{dB}$ high-to-low (2,300 and 1,100 Hz) FM sweep. The frequency sweep was extended over an intrastimulus interval (or duration) of either $275,225,175$, or $125 \mathrm{~ms}$ (one duration was used per test session). Note that for purposes of analysis, the 275- and 225-ms sweep durations were classified as 'long' and the 175- and 125-ms sweep durations were classified as 'short'. Each frequency sweep was separated by a fixed between-sequence interstimulus interval (ISI) that was always $200 \mathrm{~ms}$ greater than the intrastimulus duration in use in a given test session. Cued trials consisted of a single reversal of the background sweep (from low to high) $50 \mathrm{~ms}$ prior to the 105-db, 50-ms SES.

\section{3-Tone Oddball}

The 3-tone oddball task was administered over 3 weeks in the adult period, following FM sweep testing. Each day of 3-tone oddball testing consisted of 104 trials (cued and uncued) presented in a pseudo-random order. For a schematic of the 3-tone oddball task, refer to figure 1d. In this procedure, the background sound was a repeating sequence of 3 tones played in the following order: high frequency $(2,300 \mathrm{~Hz})$ - low frequency $(1,100 \mathrm{~Hz})$ - low frequency $(1,100 \mathrm{~Hz})$. On cued trials, the SES was preceded by $50 \mathrm{~ms}$ 
by the triplet high frequency - high frequency - low frequency $(2,300 / 2,300 / 1,100 \mathrm{~Hz})$ (the 'oddball'). The amount of time separating each tone in the sequence (the intrastimulus duration) varied across the 3 weeks, but the between-sequence ISI in each task was always $200 \mathrm{~ms}$ greater than the within-sequence intrastimulus duration being used to preserve stimulus integrity or separation. During the first week of testing, animals were tested on a $60-\mathrm{ms}$ (intrastimulus)/260-ms (interstimulus) version of the 3-tone oddball task for 5 days and then progressed to a 30-/230-ms task the second week, and finally to a 10-/210-ms task the third week. Multiple days of testing were used at each condition due to the difficulty of the task.

\section{Water Escape and Morris Water Maze}

Spatial learning was also assessed in adult male and female rats following completion of auditory testing. First, all animals were tested on a water escape task before beginning the Morris water maze (MWM). In the water escape task, the animals were required to swim a short distance in an oval metal tub ( $40.5 \times 21.5$ inches) to a visible platform ( 4 inches in diameter). The rats were released at the opposite end of the tub from the platform, and the latency to swim and climb onto the platform was recorded. The water escape task was used to rule out motivational, motor, or visual impairments as a cause for differences in the MWM performance. MWM testing began the day after water escape testing and was administered over a period of 5 days. Testing took place in a round black Plexiglas pool (48 inches in diameter). An 8 -inch submerged (invisible) platform was placed in the same position in the southeast quadrant of the tub every day of testing. Static extramaze cues were present in the room (the doorway, the light source, the experimenter, and large shapes painted black onto white walls). Both the tub and platform were painted black to eliminate intramaze cues. On each day of testing, animals underwent 4 trials on the MWM, being released from a new compass point (N, S, E, W) in the maze at the start of each trial. On the first trial on the first day of testing, each animal was placed on the platform for $10 \mathrm{~s}$ prior to being placed in the pool. The SMART video-tracking software system (Harvard Apparatus, Holliston, Mass., USA) and a ceilingmounted digital camera were used to record distance, latency, and swim speed data throughout MWM testing. A probe trial was conducted on the last day of MWM testing during which the goal platform was removed from the maze. The amount of time rats spent in the quadrant where the goal platform had been located throughout the week was recorded. This served as a measure of each animal's spatial memory for the location of the platform as well as a control for any potential olfactory or somatosensory cues provided by the submerged platform.

\section{5-Choice Serial Reaction Time Testing (Males Only)}

Following completion of MWM testing, the male subjects were tested on a 5-choice serial reaction time (5CSRT) task which measured visual attention abilities. (Note that equipment limitations allowed us to test only a subset of animals, and we chose to assess males since we have in hand more data behaviorally characterizing Dyx1c1 shRNA-transfected males. Future studies will be needed to assess attention in Dyx1c1 shRNA-transfected females.) The 5CSRT task is an operant training task that requires animals to respond to brief presentations of light stimuli with a nose poke into a lighted hole. Correct responses were rewarded with a palatable sugar pellet.
Our 5CSRT setup consisted of 3 operant chambers placed in sound and light attenuating boxes (MED-NP5L-B; Med Associates). Each operant chamber had 5 nose-poke holes on one wall and a single reward receptacle on the opposite wall. The nose-poke holes and reward receptacle were equipped with infrared beams that detected entry by a rat. An external pellet dispenser was attached to each operant chamber. Each operant chamber communicated with the attached Dell PC via the Med Associates Smart Ctrl 8 In/16 Out Package (DIG-716P2), and we used the software program Trans-IV to translate and compile custom Med State Notation programs for testing. We also used Med-PC IV to run the testing programs and acquire data.

Prior to the start of 5CSRT testing, male rats were food restricted until they had lost $10 \%$ of their body weight to ensure adequate motivation for the food reward offered during testing. Daily weight records were kept and standard lab chow was provided as necessary to ensure that the animals did not drop below $90 \%$ of their free-fed age-corrected body weight at any point during testing. The week before 5CSRT testing began, the animals were given 3 days of habituation training, which consisted of a single 20-min session in the operant chamber each day with the house light and all nosepoke and receptacle lights turned on. Reward pellets were placed in each of the 5 nose-poke holes and in the reward receptacle to familiarize the animals with the testing environment as well as the nose-poking behavior and the delivery location of sugar pellets. Following habituation, rats were given a single week of nose-poke training to gradually shape their behavior so that they would learn to respond to one lighted hole. During these 30-min sessions, the house lights in the operant chambers were turned off, all 5 nosepoke stimulus lights would light up on the first trial, and a nose poke into any of the 5 illuminated holes resulted in the delivery of a reward pellet in the reward receptacle. Based on animal response patterns, the number of lighted holes was gradually reduced down to 1 within each testing session (that is, the hole that was chosen by the animal on the previous trial did not illuminate on the next trial).

At the completion of training, 5 weeks of 5CSRT testing began. Throughout testing the stimulus duration, response interval (limited hold), and ITI (the time between the retrieval of a reward and the presentation of the next light stimulus) were gradually reduced to increase the difficulty of the task. Across 4 weeks of testing, animals progressed from a 60/60/5 program (60-second stimulus duration, 60 -second limited hold, 5 -second ITI), to a 30/30/5 program, $10 / 10 / 5$ program, and eventually to a $5 / 5 / 5$ program. The group average of percent correct responses was used to determine when all of the animals could be moved on to the next testing program. Specifically, 2 consecutive days of an average of at least $60 \%$ correct responses was used as the criterion to move on to the next testing program. During the fifth week, the rats were tested on a variable ITI task. The stimulus duration and limited hold were each set at $10 \mathrm{~ms}$ for this task. However, the ITI varied in a pseudorandom order between $2,5,10$, and $15 \mathrm{~ms}$.

The main dependent variables of interest that were measured through 5CSRT testing were the percent correct responses [calculated as the number of correct responses divided by the total number of trials including both errors and omissions (trials with no responses)] and the percent omissions (the number of trials on which the animal failed to respond in the set response window divided by the total number of trials). 


\section{Histological Preparation}

Following completion of behavioral testing, all animals (males and females) were transcardially perfused for brain analysis and identification of fluorescent proteins labels which revealed each animal's identity as either a sham or Dyx1c1 shRNA-treated animal. Before transcardial perfusion, all animals were deeply anesthetized with an intraperitoneal injection of a ketamine/xylazine mixture (100 and $15 \mathrm{mg} / \mathrm{kg}$, respectively). Rats were perfused with $0.4 \mathrm{M}$ phosphate-buffered saline followed by $4 \%$ paraformaldehyde. Heads were removed and brains were extracted and postfixed in $4 \%$ paraformaldehyde solution. Brains were shipped to G.D.R. at the Beth Israel Deaconess Medical Center for histological processing. They were cryoprotected in a $30 \%$ sucrose buffer solution before being sliced in the coronal plane at $40 \mu \mathrm{m}$ thickness. A 1-in-10 series of sections was mounted and stained for Nissl substance with thionine. An adjacent series of free-floating sections were mounted and screened for fluorescent protein labels using fluorescent microscopy to detect GFP or RFP. A final series of sections was immunohistochemically processed for visualization of RFP or GFP (Chemicon, 1:200) using ABC protocols. Light microscopy and qualitative descriptors were used to identify any visible dysplasia in sham- and shRNA-treated rats.

\section{Stereology}

Volumetric Analyses

Brains from a second cohort of male animals were used for volumetric analyses of the cortex and hippocampus and cell size analysis of the MGN. Here, a total of 28 male Sprague-Dawley shams and 29 male Sprague-Dawley Dyx1c1 shRNA-treated animals were used for volumetric reconstruction of the cortex and hippocampus. These animals had been tested on an 8-arm radial water maze working memory task, were sacrificed at a comparable age, and were found to have significant impairments on the task as compared to shams (for details, see [52]). A 1-in-10 mounted series of $40-\mu \mathrm{m}$-thick Nissl-stained sections (exactly as above) was used for analysis. Volumes of the cerebral cortex and hippocampus were measured at 2.5× magnification on an Axio 2 Zeiss Microscope using Stereo Investigator (MBF Bioscience, Williston, Vt., USA). The Cavalieri Estimator probe was used to overlay a grid of $600 \times 600 \mu \mathrm{m}$, and all points of intersection on the grid within the boundaries of the outlined region of interest were counted. Specifically, all regions dorsal to the rhinal fissure were included in the cortex measurements, including areas extending up and onto the medial surface. The hippocampal measurements included the subiculum, dentate gyrus, CA1, CA2, and CA3. These measurements, along with the section thickness and distance between each section counted, were entered into the Cavalieri equation, which yielded an estimate of the volume. Volume estimates for the right and left cortex as well as the hippocampus were calculated separately. An average of 10 sections per animal was counted for the cortex and hippocampus.

The midsagittal area of the corpus callosum was also measured for each of these animals using the Axio 2 Zeiss microscope and Stereo Investigator software. Measurements were taken at $10 \times$ magnification. The corpus callosum was analyzed on systemically sampled sections, beginning rostrally where the corpus callosum was first seen to cross the midline and continuing caudally until the last section where the corpus callosum crossed the midline. On each coronal section measured, a line was drawn along the dorsalventral axis of the brain at the midline, extending beyond the dor-

Behavioral and Anatomical Changes in Male and Female Rats after RNAi for Dyx1c1 sal and ventral boundaries of the corpus callosum, and we then measured the dorsal-ventral length of the midline of the corpus callosum. These measurements, along with the thickness of the mounted slices and the distance between each counted section, were entered into the Cavalieri equation, which yielded an estimate of the midsagittal area of the corpus callosum (for details, see [61]).

MGN Cell Size Analysis

Brains from a third cohort of like-treated and same age-of-sacrifice $D y x 1 c 1$ and sham male Wistar rats were used for MGN cell size analysis. The details of behavioral testing and histological preparation of these brains were described previously [43, 51]. Briefly, a total of 23 celloidin-embedded Nissl-stained brains were analyzed using the Axio 2 Zeiss Microscope and Stereo Investigator. An average of 4 brain sections per subject containing the MGN were chosen for analysis using a stereotaxic rat brain atlas [Paxinos and Watson, 2007]. In the Stereo Investigator, contours outlining the entire MGN were drawn at $10 \times$ magnification. Cell size measurements were taken at $100 \times$ magnification using the Nucleator probe in Stereo Investigator. Neurons were distinguished from non-neuronal cell types by the presence of a nucleolus; only neurons were measured for this analysis. A grid size of $250 \times 250 \mu \mathrm{m}$ and a counting frame of $25 \times 25 \mu \mathrm{m}$ were utilized. Neurons were only counted when their nucleolus was in sharp focus and when they were contained within the active borders of the counting frame. We obtained estimates of the neuronal area in the left MGN, right MGN, and total (right + left) MGN for each animal.

\section{Data Analyses}

Multivariate analyses of variance (ANOVA), and post hoc testing when appropriate, were used to analyze the ATT scores from auditory testing as well as the latency and swim speed measures from MWM testing between sexes and treatment groups. Repeated-measures ANOVA was used to analyze the percent correct responses and percent omissions from male visual attention testing. Repeated-measures ANOVA was also used to analyze MGN cell size distribution data between the treatment groups. Univariate ANOVA was used to analyze the cortical volume, hippocampal volume, and midsagittal area of the corpus callosum between the treatment groups. All statistical analyses of behavioral and morphological data were performed using IBM SPSS Statistics Standard Edition version 19.0. All reported p values are two-tailed unless otherwise stated.

\section{Results}

\section{Histology and Treatment Verification}

Qualitative observation of GFP and RFP in the brains from the 17 male and 18 female Wistar rats used for behavioral testing revealed 11 female sham animals, 7 female Dyx1c1 shRNA animals, 8 male sham, and 9 male Dyx1c1 shRNA animals. Further histological classification revealed 4 categories of cortical disruption within the male and female Dyx1c1 shRNA groups (see fig. 2): (1) injection site ectopia (female Dyx1c1 shRNA, $\mathrm{n}=3$; male 
Fig. 2. Coronal Nissl-stained brain sections from male (a, b) and female (c-e) Dyx1c1 shRNA animals. Histology revealed 3 categories of gross cortical malformation. a, c Injection site ectopia (female Dyx1c1 shRNA, $\mathrm{n}=3$, male Dyx1c1 shRNA, $\mathrm{n}=4$ ). These anomalies resulted from the injection puncture wound and were observed as collections of ectopic cells in layer I of the neocortex (arrows). b, d Unmigrated neurons in the white matter (female Dyx1c1 shRNA, $\mathrm{n}=5$; male Dyx1c1 shRNA, $\mathrm{n}=2$ ). These anomalies were observed as small and large collections of unmigrated cells forming heterotopic pockets in the white matter subjacent to the cortex (arrows). b,e Hippocampal dysplasia (female Dyx1c1 shRNA, $\mathrm{n}=1$; male Dyx1c1 shRNA, $\mathrm{n}=2$ ). These anomalies were observed as pockets of unmigrated cells that disrupted the structure of the hippocampal formation (most often the dentate gyrus) (arrowheads). Scale bars $=500 \mu \mathrm{m}$.

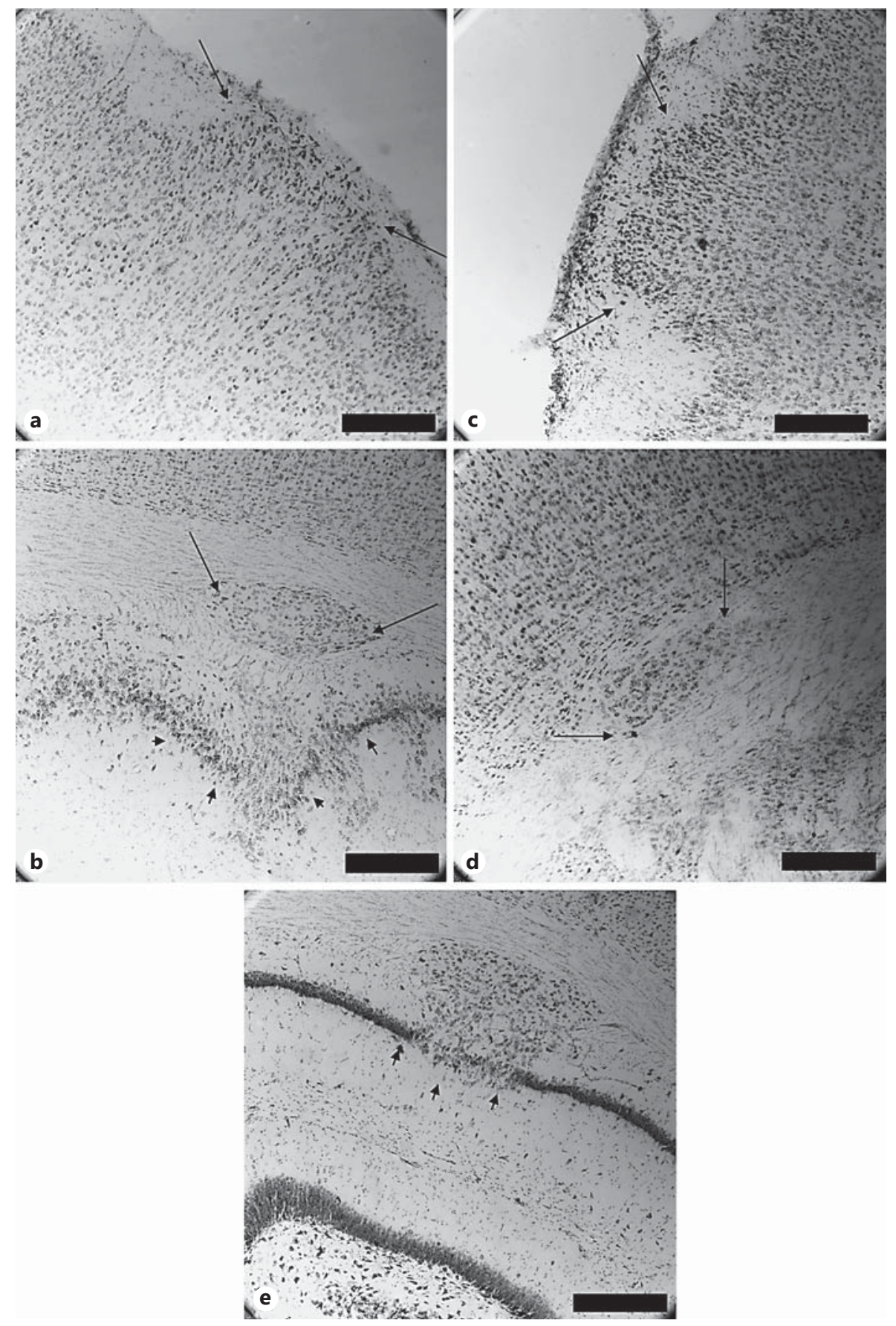

Dyx1c1 shRNA, $\mathrm{n}=4$ ), which describes an ectopic collection of cells in layer I as a result of the injection puncture wound during in utero electroporation; (2) unmigrated neurons in the white matter (female Dyx1c1 shRNA, $\mathrm{n}=$ 5; male Dyx1c1 shRNA, $\mathrm{n}=2$ ), which describes small and large collections of unmigrated cells forming heterotopic pockets in the white matter subjacent to the cortex; (3) hippocampal dysplasia (female Dyx1c1 shRNA, $\mathrm{n}=1$; male Dyx1c1 shRNA, $\mathrm{n}=2$ ), which describes pockets of unmigrated cells that disrupted the structure of the hippocampal formation (most often the dentate gyrus), and (4) no visible malformations (female Dyx1c1 shRNA, $\mathrm{n}=$ 1; male Dyx1c1 shRNA, $\mathrm{n}=4$ ), which describes any animal whose brain did not display any of the gross malfor- 

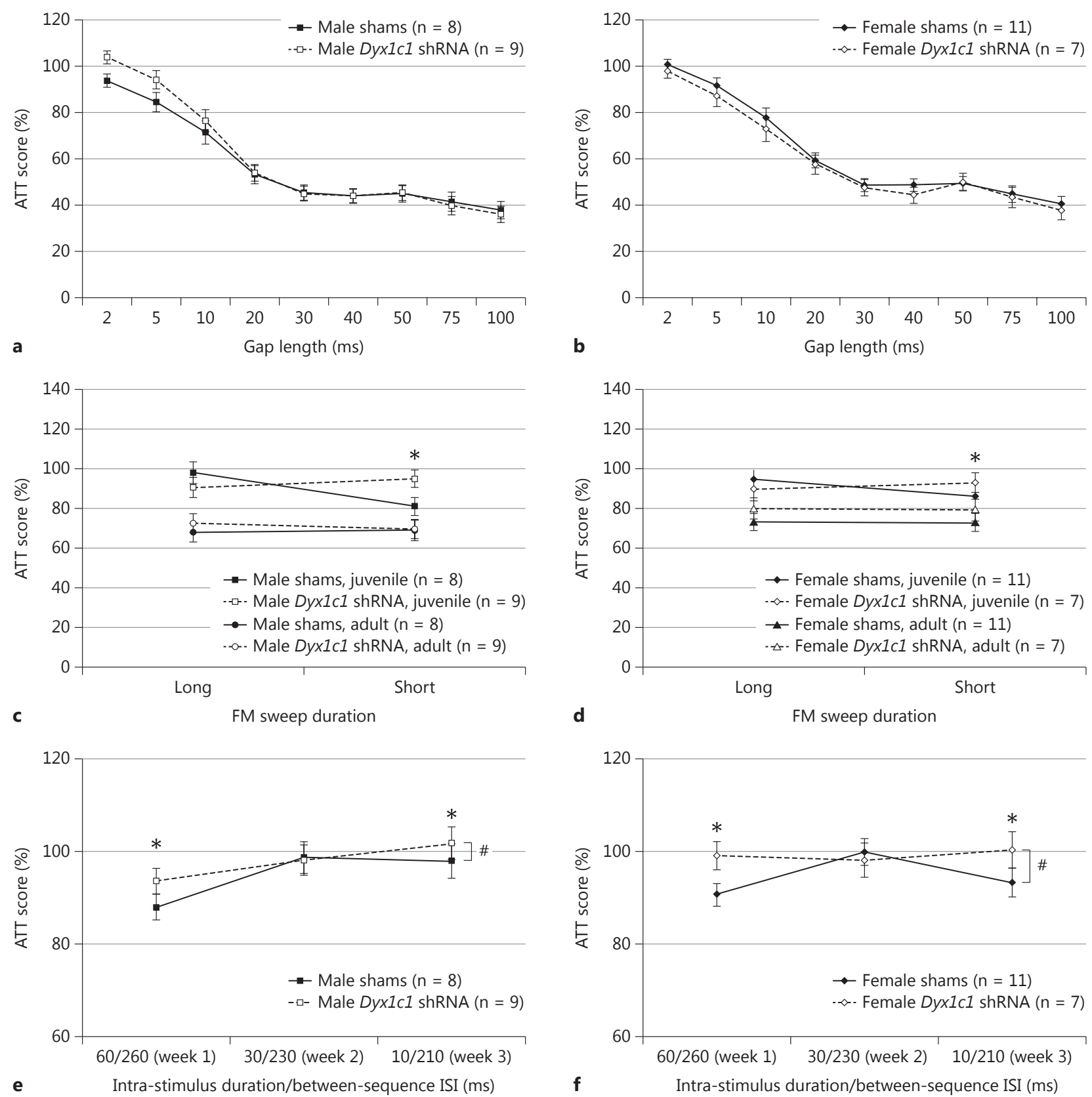

Fig. 3. Auditory testing performance by male and female Dyx1c1 shRNA and sham rats. a, b Juvenile silent gap ATT scores. These graphs illustrate similar performance between males (a) and females (b) and between sham and Dyx1c1 shRNA animals. Pairedsamples t tests indicated that animals could successfully detect gaps down to $5 \mathrm{~ms}$ in length. c, d FM sweep ATT scores. These graphs illustrate FM sweep performance by males (c) and females (d) in the juvenile period and adulthood. A significant age $\times$ sweep duration $\times$ treatment interaction indicates that at the short sweep duration, sham animals consistently perform better than Dyx1c1 animals (as indicated by their lower ATT scores), across both age and

sex $(* p<0.05)$. e, $\mathbf{f} 3$-Tone oddball ATT scores. These graphs illustrate the 3 -tone oddball performance by males (e) and females (f) in the adult period. ATT scores were averaged across 5 days for each of the 3 versions of this task. There was a marginal effect of treatment across the 3 tasks suggesting worse overall performance by Dyx1c1 shRNA animals as compared to shams (across sex) $\left({ }^{\#} \mathrm{p}=0.09\right)$. Separate analyses revealed significant treatment effects on the $60 / 260$ and $10 / 210$ versions of the task $(* p<0.05)$, with shams outperforming Dyx1c1 shRNA animals across sexes. Values represent means \pm SEM. 
mations described in the other categories. The malformations varied in size and number, and many subjects ( $\mathrm{n}=$ 12) displayed multiple types of disruption. The male and female Dyx $1 c 1$ shRNA brains appeared qualitatively similar, with the majority of animals in each case displaying at least one form of disruption. It is worth noting that, during in utero electroporation surgery, the copper alloy paddles were placed in such a way as to primarily target transfection of cell populations destined for regions of the parietal cortex, as has been done in all of our previous RNAi studies (for more technical details, see [62]).

Observation of tissue sections stained for RFP and GFP confirmed that the vast majority of labeled cells in each animal were present in the parietal cortex. Note that in addition to being analyzed as a function of sex and treatment (Dyx1c1 shRNA vs. sham), all behavioral results were also analyzed as a function of these histological subgroups. However, a significant main effect of the histological category was not found, and so data are only presented here as a function of sex and treatment groups.

\section{Auditory Testing}

Normal Single Tone

Paired-samples $t$ tests were used to compare the mean uncued and cued startle response amplitude for all subjects on the normal single tone task. Analyses revealed significant attenuation on cued trials in both male and female sham and Dyx1c1 shRNA animals $(\mathrm{p}<0.05)$. A univariate ANOVA with sex (2 levels) and treatment (2 levels) showed no main effect of sex and treatment, nor a sex $\times$ treatment interaction $[F(1,31)<1$, n.s. in all cases $]$ on the mean ATT scores for the task. These results indicate that there were no differences between the sexes or the treatment groups in basic auditory discrimination abilities or baseline prepulse inhibition.

\section{Silent Gap Detection}

Analysis of mean cued and uncued startle response amplitudes revealed that all animals were able to successfully detect gaps down to $5 \mathrm{~ms}$. A two-between (sex and treatment), two-within [day (4 levels) and gap (9 levels)] ANOVA revealed no main effect of sex and treatment, nor a sex $\times$ treatment interaction $[F(1,31)<1$, n.s. in all cases], indicating similar gap detection abilities between the sexes and treatment groups (fig. 3a, b). Significant day $[F(3,93)=126.3, \mathrm{p}<0.001]$ and gap $[F(8,248)=509.3$, $\mathrm{p}<0.001$ ] effects indicated that the performance of all animals improved across gaps of increasing length and across days of testing. There was a significant gap $\times \operatorname{sex} \times$ treatment interaction $[F(8,248)=2.1, \mathrm{p}<0.05]$, but post hoc testing revealed that this effect was due to a difference between the male Dyx1c1 shRNA group and male sham group at the 2 -ms gap only $[F(1,15)=5.2, \mathrm{p}<0.05$, with Dyx1c1 shRNA animals performing worse than shams]. Given that the paired-samples $t$ tests indicated that the animals could only significantly detect down to gaps that were $5 \mathrm{~ms}$ in length, this finding has limited implications.

\section{FM Sweep}

FM sweep performance was analyzed across age, sex, and treatment. A two-between (sex and treatment), twowithin [age (2 levels) and sweep duration (2 levels)] ANOVA revealed no significant main effects of treatment and sex, nor a sex $\times$ treatment interaction $[F(1,31)<1$, n.s. in all cases]. A significant age effect $[F(1,31)=102.8$, $\mathrm{p}<0.001]$ indicated improved performance in all adult animals as compared to juveniles. A significant sweep duration $\times$ treatment interaction was found $[F(1,31)=4.7$, $\left.\mathrm{p}<0.05, \eta_{\mathrm{p}}^{2}=0.132\right]$, indicating that at the short sweep duration shams consistently performed better than Dyx1c1 shRNA animals for both sexes and at both ages (fig. 3c, d). Finally, we found a significant age $\times$ sweep duration $\times$ treatment effect $[F(1,31)=11.7, \mathrm{p}<0.01]$. When values were assessed for the juvenile short FM condition only, we found a significant treatment effect across sexes $\left(\mathrm{p}<0.03, \eta_{\mathrm{p}}{ }^{2}=0.151\right)$, but this effect was not quite significant in adults only (although a trend was present, with Dyx1c1 shRNA animals performing worse than shams).

\section{3-Tone Oddball}

ATT scores from each version of the 3-tone oddball task were averaged across days for each rat, resulting in one mean ATT score for the 60-/260-ms version of the task, one for the 30-/230-ms version, and one for the 10-/ 210 -ms version (per animal). These ATT scores were analyzed in a two-between (sex and treatment) and onewithin [task (3 levels)] ANOVA. We did not find a main effect of treatment across the 3 tasks, although a trend was present $\left[F(1,31)=2.9, \mathrm{p}=0.10 \eta_{\mathrm{p}}{ }^{2}=0.086\right]$, with $D y \times 1 c 1$ shRNA-treated animals having slightly higher average ATT scores than shams on all 3 versions of this task (fig. 3e, f). There was not a main effect of sex nor a sex $x$ treatment interaction $[F(1,31)<1$, n.s. for both effects]. A significant within-subjects effect of task $[F(2,62)=5.1$, $\mathrm{p}<0.01$ ] indicated that each group performed worse as the ISI used within the task decreased (and thus the task increased in difficulty). Individual one-within [day (5 levels)] and one-between [treatment (2 levels)] ANOVAs on each of the tasks revealed a significant treatment effect on
Szalkowski/Booker/Truong/Threlkeld/ Rosen/Fitch 
Fig. 4. MWM data. a The average latency to mount a visible platform during the water escape task, given the day before the start of MWM testing. There were no main effects of sex or treatment, indicating that male and female Dyx1c1 shRNA and sham animals were all able to see, swim, and mount the platform. $\mathbf{b}$ The average time spent in each of the 4 maze quadrants during the probe trial. All animals spent significantly more time in the target quadrant during the probe trial (indicated by ** $\mathrm{p}<0.01$ ), which indicates that all animals successfully learned the location of the goal platform across the 4 prior days of testing. There were no main effects of sex or treatment on the probe trial data. c MWM average path length to the platform. A significant effect of $\operatorname{sex}(* \mathrm{p}<0.05)$ indicates that females swam longer paths than males before reaching the platform across 4 days of testing. No main effect of treatment was found, indicating that sham and Dyx1c1 shRNA animals swam similar distances before finding the goal platform. Values represent means \pm SEM.

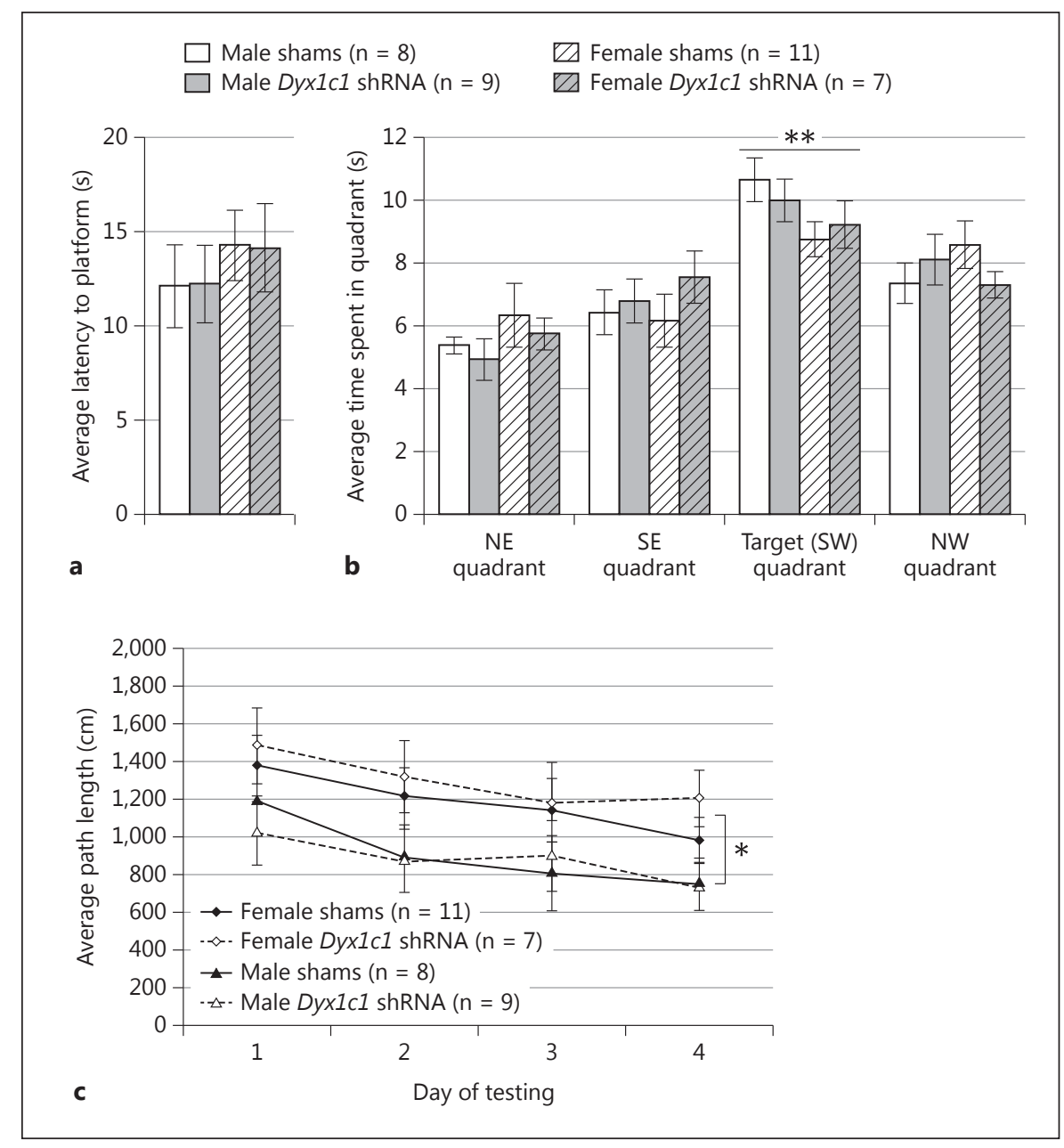

the 60-/260-ms version of the task $[F(1,29)=5.9, \mathrm{p}<0.05$, $\left.\eta_{\mathrm{p}}{ }^{2}=0.168\right]$ and a significant day $\times$ treatment interaction on the $10-/ 210-\mathrm{ms}$ version of the task $[F(4,124)=4.0$, $\mathrm{p}<0.01, \eta_{\mathrm{p}}{ }^{2}=0.113$ ], indicating that Dyx1c1 shRNA subjects performed worse than shams across sexes.

\section{Water Escape and MWM}

Latency to mount the platform during the water escape task was analyzed using a two-between (sex and treatment) ANOVA (fig. 4a). We found no main effect of sex and treatment, nor a sex $\times$ treatment interaction $[F(1,31)$ $<1$, n.s. in all cases], indicating that there were no baseline differences in the animals' ability to see, swim, or mount the platform. For the MWM data, a two-between (sex and treatment), one-within (day) ANOVA compared the average swim speed across all 4 trials throughout the 4 days of testing. This analysis revealed no significant main effects of sex and treatment, nor a sex $\times$ treatment interac- tion $[F(1,31)<1$, n.s. in all cases]. There was, however, a significant within-subjects day $\times$ sex interaction $[F(3$, $93)=3.0, p<0.05]$, which was due to the fact that male rats swam significantly faster than female rats on the last 2 days of testing.

To assess differences in spatial learning, a two-between (sex and treatment), one-within (day) ANOVA was carried out to compare the average length of the swim path to reach the platform between groups across 4 trials per day and 4 days of testing. The analysis revealed a significant main effect of sex $[F(1,31)=8.2, \mathrm{p}<0.01]$ due to the fact that, across treatment, females swam significantly longer paths to the goal platform than males (fig. 4c). This finding is not surprising given the well-documented evidence of differences in spatial navigation abilities between male and female rodents (for a review, see [63]). There was neither a main effect of treatment nor a significant sex $\times$ treatment interaction $[F(1,31)<1$, n.s. in 


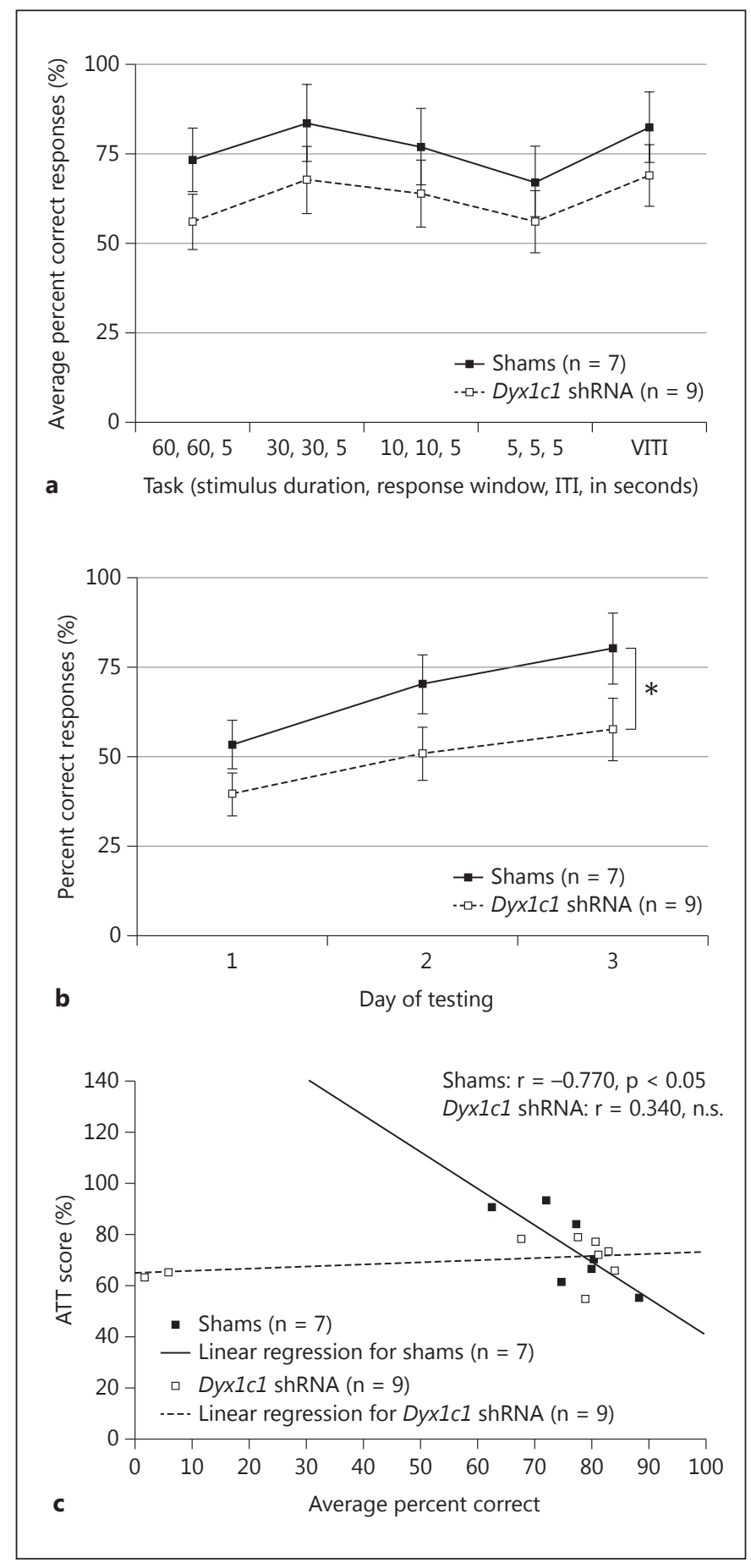

Fig. 5. Visual attention testing performance by male Dyx1c1 shRNA and sham rats. a Overall percent correct responses as a function of visual attention task. Note that Dyx1c1 shRNA animals had consistently lower percent correct responses than sham animals, although this difference did not reach statistical significance. VITI $=$ Variable intertrial interval. $\mathbf{b}$ Average percent correct responses across 3 days of testing on the $60,60,5$ version of the 5CSRT task (60-second stimulus duration, 60-second response window, 5-second ITI). A repeated measures ANOVA revealed a both cases], indicating that Dyx1c1 shRNA rats performed the task at a level similar to shams. The only significant within-subjects effect was a day effect $[F(3,93)=$ $4.4, \mathrm{p}<0.01]$, indicating that all animals improved on the task across days of testing. For the probe trial data, a repeated-measures ANOVA was carried out to compare the average time spent in each quadrant and the average distance swam in each quadrant between groups (fig. 4b). For each analysis, there was no main effect of sex and treatment, nor a sex $\times$ treatment interaction $[F(1,31)<1$, n.s. in all cases]. There was a significant within-subjects effect of quadrant $[F(3,93)=15.0, \mathrm{p}<0.001]$, and pairwise comparisons revealed that significantly more time was spent $(\mathrm{p}<0.01)$ and longer total distances were swam (data not shown, $\mathrm{p}<0.01$ ) in the target quadrant as compared to each of the other quadrants (a Bonferroni correction for multiple testing was used). This indicates that the animals, across sex and treatment group, did indeed learn and remember the spatial location of the goal platform across 4 days of MWM testing.

\section{CSRT Testing (Males Only)}

Due to changes in task demand and parameters, performance (\% correct) was assessed at each phase separately (but overall scores are shown in fig. 5a, with Dyx1c1 shRNA animals consistently performing worse than shams). Individual one-within [day (5 levels)] and onebetween [treatment (2 levels)] ANOVAs were used to analyze the data from each visual attention task. A significant treatment effect was found when examining performance specifically on the $60 / 60 / 5$ version of the task, with Dyx1c1 animals performing significantly worse on the first 3 days $\left[F(1,14)=3.1, \mathrm{p}=0.05\right.$, one-tailed, $\eta_{\mathrm{p}}{ }^{2}=$ 0.182 ) (fig. 5b). A one-tail test was used based on human evidence that anomalies in the DYX1C1 gene are associated with deficits on tasks requiring sustained attention [45-49] providing a strong a priori basis to examine treatment differences in one direction only.

significant effect of treatment, with Dyx1c1 shRNA animals performing significantly worse than shams ( $* \mathrm{p}=0.05$, one-tailed). c Bivariate correlation between visual attention testing performance and rapid auditory testing scores. Shams demonstrated a significant correlation between average percent correct during visual attention testing (averaged across all tasks) and average ATT scores on adult short FM sweep testing $(\mathrm{p}<0.05)$. Dyx1c1 shRNA animals' performances on the 2 tasks were not significantly correlated. Values represent means \pm SEM. 
In order to determine if the observed deficits in attention were related to the previously observed complex acoustic processing deficits, separate bivariate correlations were run on percent correct averaged across all visual attention tasks and average adult short FM ATT scores for $D y x 1 c 1$ and sham animals (fig. 5c). The analysis revealed a significant correlation between ATT scores and percent correct for sham rats $(r=-0.77, \mathrm{p}<0.05)$, which indicates that lower ATT scores (which indicate better performance) are correlated with higher percent correct scores. However, there was no significant correlation between ATT scores and percent correct for Dyx1c1 shRNA animals ( $r=0.34$, n.s.). The significant correlation seen in shams may reflect an index of general good performance across tasks (i.e., separating 'smarter' rats on both tasks). However, importantly, the lack of a significant correlation in knockdown animals indicates that these measures are orthogonal in Dyx1c1 rats. This suggests that deficits in processing complex stimuli are independent of underlying deficits in attention; however, causal relationships cannot be drawn from correlational data.

\section{Stereology}

Volumetric Analyses

Data from the volumetric analyses of the prepared brain sections from the second cohort of male brains from Szalkowski et al. [52] were analyzed with one-way ANOVAs. No significant effect of treatment was found for the midsagittal area of the corpus callosum, left or right cortex volume, or left or right hippocampus volume $[F(1,55)<1$, n.s. in all cases]. All of the current stereological measures were analyzed as a function of the presence or absence of visible malformations in Dyx1c1 shRNA animals, and no significant differences in the main effect of visible malformation was found.

\section{MGN Cell Size Analysis}

Note that measures from the right and left MGN were analyzed separately using hemisphere as a repeated measure, but no effect of hemisphere nor any interactions were found and so the data reported here are summed over this variable. A one-way ANOVA revealed that there was no significant treatment effect on the average total MGN neuron size, average left MGN neuron size, or average right $M G N$ neuron size $[F(1,21)<1$, n.s. in all cases]. For total MGN neuronal size distribution analysis, we computed a frequency distribution using 14 consecutive bins ranging from $0-230 \mu \mathrm{m}^{2}$. The number of neurons in each bin was converted to a percentage of the total num-

Behavioral and Anatomical Changes in Male and Female Rats after RNAi for Dyx1c1 ber of neurons counted for each animal to control for differences in the number of cells counted between animals. To compare the distribution of cells across the 14 bins between groups, a series of 14 one-between (treatment), one-within [bin (13 levels)] ANOVAs were carried out, with a different bin being excluded from each analysis. Average $\mathrm{F}$ values were computed for within- and between-subjects comparisons and were compared to criti$\mathrm{cal} \mathrm{F}$ values in a table using the appropriate degrees of freedom to determine significance. This analysis revealed no significant main effect of treatment $[F(1,21)=1.4$, n.s.]. There was, however, a significant within-subjects effect of bin $[F(13,273)=44.9, \mathrm{p}<0.001]$ as well as a significant bin $\times$ treatment interaction $[F(13,273)=1.8, \mathrm{p}=$ $\left.0.05, \eta_{\mathrm{p}}{ }^{2}=0.077\right]$. The significant interaction indicated that Dyx1c1 shRNA-treated animals had significantly more neurons in the smaller-size bins as compared to their sham-treated counterparts (fig. 6). To verify the significance of this difference in the data in another way, we also performed a two-sample Kolmogorov-Smirnov test to compare non-binned distributions of the raw MGN neuronal size data between the Dyx1c1 shRNA animals and the sham animals. This test also yielded a significant result ( $\mathrm{p}<0.001)$, further illustrating that there are significantly more small cells (and fewer large cells) in Dyx1c1 shRNA-treated animals. Note that we ran bivariate correlations on between the average MGN cell size data for Dyx1c1 shRNA-treated animals and sham animals and their corresponding auditory data from a previous study [51]. However, these correlations did not yield significant results (possibly due to the small group sizes: Dyx1c1 shRNA $\mathrm{n}=16$ and sham $\mathrm{n}=9$ ), and so we do not report them here.

\section{Discussion}

We found that knocking down Dyxlcl expression in a subset of neocortical cells in the developing rat brain leads to disrupted neuronal migration and was associated in the current study with specific impairments on complex acoustic processing tasks in both male and female rats, with no effects on simple spatial learning and memory (MWM). Early interference with $D y \times 1 c 1$ was also found to lead to visual attention deficits in male rats, and these deficits appear to be orthogonal to the auditory processing deficits (based on a lack of correlation). Analyses of gross brain morphology revealed that early interference with Dyx1c1 did not lead to changes in the midsagittal area of the corpus callosum, volume of the cortex or vol- 
Fig. 6. Cell size distribution in the MGN of the thalamus. This frequency histogram represents the distribution of cell size in the MGN of Dyx1c1 shRNA (black bars) and sham (white bars) animals. Note that only male brains were analyzed. The average percent of cells in each size bin for each treatment group was calculated for 14 consecutive bins based on the cell area. A significant bin $\times$ treatment interaction $(\mathrm{p}=$ 0.05 ) indicates that Dyx1c1 shRNA animals have more small MGN neurons than their sham counterparts. Post hoc testing revealed that the biggest contributor to this interaction was a significant difference at the fourth size bin, where the Dyx1c1 shRNA animals had significantly more cells than shams $\left({ }^{*} \mathrm{p}<0.05\right)$.

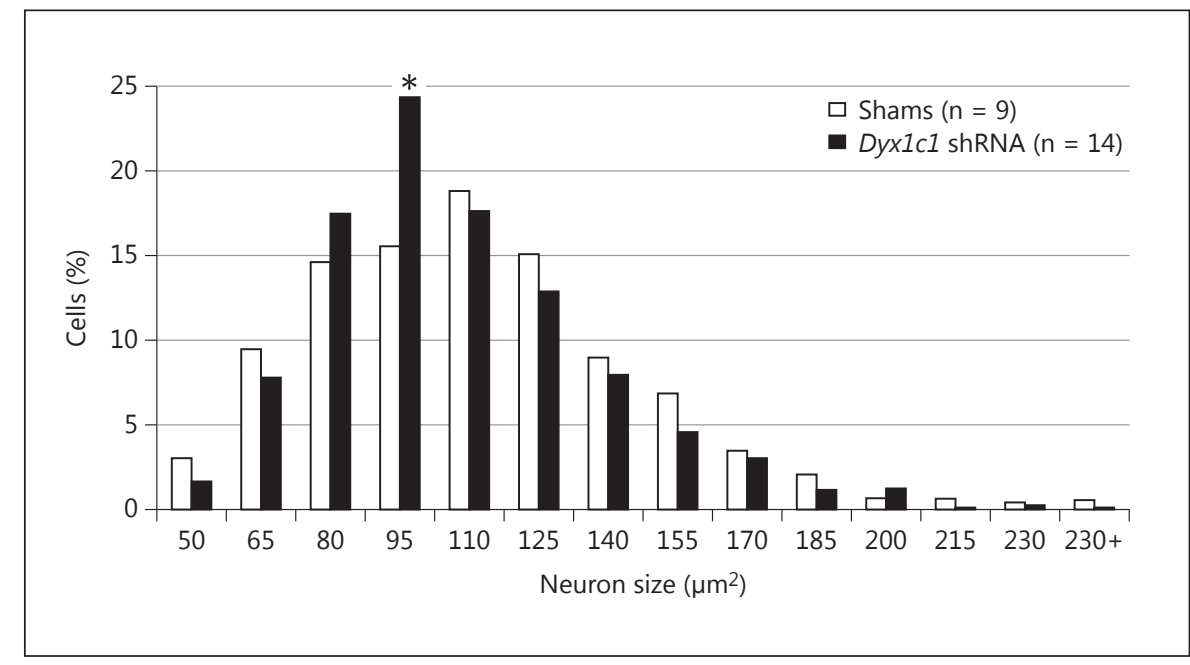

ume of the hippocampus. However, Dyx1c1 shRNA animals did exhibit a shift in the distribution of neuron sizes in the MGN of the thalamus, with more small and fewer large neurons as compared to sham counterparts. The current study thus provides additional support for the role of Dyx1c1 in disrupting behavioral processes that parallel those associated with dyslexia in humans as well as for a role for Dyx1c1 in neuronal migration and more widespread developmental brain circuitry.

\section{Dyx1c1 RNAi and Behavior: Auditory Processing}

Deficits in processing rapidly presented verbal and non-verbal acoustic information have been shown in many individuals with dyslexia and other forms of developmental language impairment [12, 15, 39, 64-67]. Moreover, it has been suggested that rapid auditory processing skills underlie in part higher cognitive processes such as phonological processing (which plays an essential role in reading acquisition) in both dyslexic and non-dyslexic populations [68-70]. Importantly, the current study replicated findings reported in Threlkeld et al. [51], specifically that $D y \times 1 c 1$ knockdown leads to subsequent impairments on a complex auditory processing task as seen in both juvenile and adult male animals (though effects here are more pronounced at the juvenile age, consistent with prior evidence that experience can ameliorate some deficits [71]). In both the current study and the Threlkeld et al. [51] study, rapid auditory processing deficits were found in spite of the fact that the primary auditory cortex was neither directly transfected nor visibly disrupted as a result of the RNAi procedure. We hypothesize that the observed auditory processing impairments result because early disruption of cortical neuronal migration has widespread effects on cortical organization that are not obvious in gross neuroanatomical analysis (i.e., Nissl stain). This hypothesis is supported by the observation that the cell size distribution in the MGN shifts following these developmental perturbations (fig. 6). The MGN (which is a neural substrate of audition) receives reciprocal connections from the primary auditory cortex. The fact that the morphology of this nucleus changes in the absence of gross morphometric disruptions to the primary auditory cortex suggests that there are widespread changes to cortical organization following these 'focal' disruptions of neuronal migration.

The current study also demonstrated that disruption of Dyx1c1 lead to equivalent acoustic processing deficits in male and female animals. This latter finding might appear in stark contrast to previous reports demonstrating a female advantage following other types of early brain disruption in rats $[53,55,72]$. However, whereas prior reports emphasize sex differences in response to damage, the cascade of brain changes following Dyx1c1 knockdown may not specifically trigger apoptotic events and thus may be independent of sex or sex-specific hormonal pathways shown to modulate apoptosis [73]. One study from our lab demonstrated a female advantage following cortical dysgenesis in an animal model that did not involve injury per se, using a strain of mice that exhibit spontaneously occurring layer I ectopia [54]. In this study, it is important to note that genetic factors were not assessed, but rather physical evidence of disruption was used to categorize affected and unaffected male and female mice for statistical comparison. It is unclear what 
sex effects might be seen in the BXSB mice if groups could be divided by genotype rather than cortical characterization, especially since we have subsequently shown that genotype and not presence or absence of disruption is the key variable in determining behavioral deficits. However, the gene modulating the presence of ectopias in the BXSB mouse strain has not yet been identified, and therefore it is not possible to analyze behavioral data in male and female BXSB mice as a function of genes instead of malformations. For this reason, caution must be used in comparing sex differences across these two studies. Interestingly, some recent studies have indicated that the Dyx1c1 protein may be associated with alpha and beta estrogen receptors in the brain during development, and that this association may have important sex-dependent implications for the role of this protein in brain development [74]. However, this study exclusively examined the deleterious effects of overexpression of Dyx $1 c 1$ on estrogen receptor signaling, and so it is difficult to interpret that theory in the context of reduced Dyx1c1 expression. Ultimately, the dramatic sex differences in diagnostic ratios for dyslexia (estimated at $1.5: 1$ to $3: 1$ [1]) remain to be explained.

\section{Dyx1c1 RNAi and Behavior: Spatial Memory}

It is important to note that, in the current study, Dyx1c1 knockdown did not lead to spatial memory deficits on the MWM task. Similarly, in the Threlkeld et al. [51] study, spatial memory deficits were only observed in Dyx1c1 shRNA animals with specific disruption to the hippocampus, suggesting that if the hippocampus is not directly disrupted, Dyx1c1 RNAi does not lead to simple spatial memory impairments. In our study, only 3 animals exhibited disruption of the hippocampus, and therefore this statistical comparison could not be made. Still, the overall Dyx1c1 group showed no deficits in simple spatial memory. This result may seem paradoxical given our previous report that Dyx1c1 shRNA-treated animals exhibit deficits in spatial working memory (as assessed on an 8-arm radial water maze task) [52]. However, the radial arm water maze may tap into a neural circuit that is different than hippocampus-dependent spatial navigation abilities. Thus, embryonic Dyx1c1 shRNA transfection appears to detrimentally affect spatial working memory even though simple spatial memory and navigation abilities remain intact. Importantly, there was a significant sex effect for both Dyx1c1 shRNA and sham females, which performed the MWM more poorly than their male counterparts. This result is consistent with documented sex differences in spatial navigation abilities [63]. More-

Behavioral and Anatomical Changes in Male and Female Rats after RNAi for Dyx1c1 over, the ability of transfected animals to learn and perform a task of simple spatial memory indicates that the Dyx $1 c 1$ genetic disruption does not create a global deficit in learning and cognitive ability. Instead, the observed impairments are subtle and domain specific. These findings run nicely in parallel to effects observed in dyslexic populations, with dyslexic individuals demonstrating significant impairments in reading despite otherwise normal IQ levels.

\section{Dyx1c1 RNAi and Behavior: Visual Attention}

In addition to auditory and phonological impairments, deficits in visual attention have also been reported in some dyslexic individuals [10,75-77]. In fact, one recent study demonstrated that performance on a visual attention task was a better predictor of future reading ability than other language-related scores among a sample of kindergarteners [10]. Interestingly, some recent evidence has suggested that these visual attention deficits and phonological deficits may be orthogonal to one another and, moreover, likely mediated by separate neural mechanisms $[4,78]$. Additional support for the role of attention in reading comes from the high comorbidity between dyslexia and ADHD, and the observation that $40 \%$ of children diagnosed with ADHD also exhibit reading impairments (although this overlap may not be due to deficits in visuospatial attention specifically) [79-81]. In the current study, knocking down Dyx1c1 led to significant impairments in visual attention in male rodents. To the best of our knowledge, this is the first report of visual attention deficits in an animal model following knockdown of Dyx1c1 (or any CDSG). As with the auditory processing data, deficits found in visual attention in Dyx1c1 shRNA rats were significant but subtle, in fact suggesting that ongoing experience can ameliorate some of the deficits seen at earlier ages and stages of testing.

It is also interesting to note that the attention deficit appears to be orthogonal to the auditory processing deficit. Specifically, while we found a significant correlation between high performance on rapid auditory processing tasks and high performance on visual attention tasks in shams, there was no correlation between rapid auditory processing scores and visual attention scores in Dyx1c1 shRNA animals. We hypothesize that this is due to the fact that the two behavioral domains tested have semiindependent underlying neural controls, which could support each other in a normal system (i.e., to produce 'high performers'). Developmental disruption may specifically disassociate from these systems by differentially impacting underlying neural systems of attention and 
acoustic processing. However, deficits in rapid auditory processing abilities are likely mediated by alterations in sensory processing pathways within the brain (such as those involving the MGN, which we have shown to be disrupted in this model), while deficits in visual attention may be mediated by disruptions to higher-order core executive functioning systems. Executive functioning abilities include other domains such as short-term and working memory, which has also been shown to be deficient following Dyx1c1 shRNA transfection in rats [52]. Future studies will look at potential correlations between working memory abilities and visual attention in Dyx1c1 shRNA-transfected rats to explore this possibility.

\section{Dyx1c1 RNAi and Brain Morphology}

Several reports have come out within the last 5 years to support the view that Dyx1c1 plays a role in neuronal migration in the brains of rodents [43, 44, 51, 52, 82].

This evidence is striking, given the observation of neuronal migration disorders in the brains of human dyslexic patients [16]. Across all studies, several common categories of brain disruption have been observed in rats following Dyx1c1 RNAi. These include the following: heterotopia or unmigrated neurons in the white matter; injection site ectopias, which are collections of cells in layer I of the neocortex, resulting from the injection puncture wound; hippocampal dysplasia, in which collections of unmigrated neocortical cells disrupt regions of the hippocampal formation, and, occasionally, non-injection site ectopias, in which collections of neocortical cells overmigrate into layer I of the neocortex distal from the injection site. While the exact biological function of the Dyx1c1 protein is still being studied, some studies have suggested that it plays a role in cytoskeletal dynamics, which are crucial for allowing cells to adapt and change shape during the process of migration [44]. Another commonality among the rat RNAi studies of Dyx $1 c 1$ is the wide variability in the size and number of malformations present in each animal, in spite of similarly-sized populations of transfected cells. Interestingly, attempts to draw correlations between the behavioral impairments observed in these animals and the types and degree of cortical dysgenesis present have been inconclusive. In fact, a previous report from our lab has reported that Dyx1c1 shRNA-transfected male animals without any visible malformations performed just as poorly on a working memory task as those with one or more frank disruptions to the cortex and white matter [52]. In the current study, there were not enough animals without visible malformations to make a similar, meaningful comparison. Yet importantly, these findings stand in contrast to previous work from our lab that has demonstrated direct relationships between the degree of behavioral impairment and the degree of cortical disruption in teratogenic and injury-induced disruptions of neuronal migration [71,83]. This discrepancy may reflect the fact that subtle reorganization of the underlying circuitry may parallel the degree of injury when disruption is externally induced (e.g., by focal freezing lesion on P1 to create microgyria), but when alterations in the circuitry are the product of genetic anomalies regulating brain development, then the presence or absence of 'visible' malformations may represent more of an epiphenomenon that is not necessarily causal (but rather an orthogonal end point). This means that more finegrained assessments of cortical disruption (optical imaging, electrophysiology) may be needed to find the 'neural signature' associated with these behavioral deficits. Taken together, this suggests that the loss of Dyx1c1 protein impacts the brain differently than other types of induced migration disruption, and instead may affect the developing brain - and ultimately behavior - at a microscopic or synaptic level. Additional support for this theory comes from other studies that have reported that knocking down Dyx1c1 through RNAi impacts cells that were not directly transfected with shRNA, often leading to aberrant migration and placement of otherwise normal cells $[43,44]$. However, the exact mechanism underlying these various effects of $D y \times 1 c 1$ knockdown remains unknown.

The current study also sought to better characterize the widespread effects of Dyx1c1 knockdown on brain morphology in male rats. We found that Dyx1c1 knockdown did not cause significant changes in the midsagittal area of the corpus callosum, cortex volume, or hippocampal volume. Several neuroimaging reports in the clinical literature have demonstrated decreases in cortical gray matter and increases in corpus callosum size in dyslexic individuals as compared to unaffected controls [19-24]. However, specific relationships between Dyx1c1 variants and white matter structure have not yet been explored clinically. One recent report investigated specific relationships between changes in gray matter and variants of the CDSGs Dyx1c1, Kiaa0319, and Dcdc2 in schizophrenic humans. In that sample, $D C D C 2$ variants, and not DYX1C1 variants, were found to associate with cortical gray matter changes [49]. Thus, it is possible that previously observed changes in gross cortical and callosal size may be due to the influence of other CDSG variants, or to an as yet undiscovered mechanism. This lack of gross an- 
atomical changes in the cortex, corpus callosum, and hippocampus following Dyx1c1 knockdown further suggests that this genetic disruption changes the developing brain at a microscopic (circuitry) level.

We also explored the effects of Dyx1c1 knockdown at the cellular level in the auditory nucleus of the thalamus, the MGN, in a subset of male animals. We specifically chose to examine the MGN based on its known role in auditory processing and based on the report by Galaburda et al. [17] demonstrating a significant change in cell size distribution in the MGN of dyslexic individuals as compared to controls. Moreover, similar changes in MGN morphology have been reported in other animal models of developmental cortical dysgenesis [84-86]. In the current study, we observed a significant change in the MGN cell size distribution in Dyx1c1 shRNA-transfected animals. Specifically, Dyx1c1 shRNA animals had more small cells in the MGN than their sham-treated counterparts - a shift that is strikingly similar to previously reported MGN changes in both animal and human studies. This was an especially intriguing finding given that this subset of animals demonstrated significant impairments in auditory processing abilities [51]. As a subcortical structure, the MGN is not directly impacted by the transfection procedure, which specifically targets neocortical neurons at the ventricular zone surface for $D y \times 1 c 1$ knockdown. Moreover, the cells transfected during in utero electroporation were not destined for the primary auditory cortex, which shares reciprocal projections with the MGN, but instead were cells that were precursor neurons of the parietal cortex (for further histological details, see [43]). Thus, it seems unlikely that the changes in the MGN were secondary to changes in immediate (primary) cortical targets. Rather, previous studies have proposed that MGN cell size distribution shifts like this one may occur as a result of atypical maintenance of transient developmental connections between the MGN and areas of non-auditory cortices following developmental brain injury [84].

Functionally, a reduction in the number of large cells in the MGN may result in impairments in processing rapid acoustic information [87]. However, we cannot draw any conclusions about causal relationships between disrupted MGN morphology and rapid auditory processing deficits in the current study. While further testing is necessary to explore this and other possibilities, it is plausible that Dyx1c1 knockdown may affect behavior through subtle and pervasive secondary subcortical changes as demonstrated here.

\section{Conclusions}

We report here that in utero $D y x 1 c 1$ knockdown led to disruptions of neuronal migration in the brains of male (and female) Wistar rats. Further, stereological investigation of transfected male rat brains revealed that this genetic disruption did not result in significant changes in the volumes of the cortex or hippocampus, nor in the midsagittal area of the corpus callosum. Transfection did, however, lead to a significant shift in cell size distribution within the MGN of male rat brains, which may have important functional implications in the domain of complex auditory processing. We further replicated previous reports of specific complex acoustic processing deficits in Dyx1c1 shRNA-transfected males and provide new evidence that this genetic disruption leads to similar behavioral impairments in females. We also found, for the first time, that Dyx1c1 shRNA transfection is associated with visual attention deficits in adult male rodents. Taken together, these results provide further support for the role of Dyx1c1 in brain development, and specifically for behaviors that parallel those often impaired in developmental dyslexia. Future studies will further investigate similarities and differences in the effects of these genetic disruptions - using this and other rodent homologs of CDSGs - in males and females, for example by testing female abilities in working memory and visual attention. Potential sex differences in gross morphological measures in the brains of transfected animals will also be explored in future work. The identification of potential gene-behavior relationships in dyslexia - as well as potential sex-specific gene-behavior relationships - could culminate in more accurate and earlier diagnoses as well as in the implementation of individually tailored early intervention in at-risk populations, ideally leading to improved cognitive outcomes for those impacted by the disorder.

\section{Acknowledgements}

This work was supported by the National Institutes of Health Grant HD057853. The authors wish to thank Dr. Joseph LoTurco for his assistance in the preparation of the plasmids and animals used in the study.

\section{Disclosure Statement}

None of the authors have a conflict of interest to declare. 


\section{References}

1 Peterson RL, Pennington BF: Developmental dyslexia. Lancet 2012;379:1997-2007.

-2 Kovelman I, Norton ES, Christodoulou JA, Gaab N, Lieberman DA, Triantafyllou C, Wolf M, Whitfield-Gabrieli S, Gabrieli JD: Brain basis of phonological awareness for spoken language in children and its disruption in dyslexia. Cereb Cortex 2012;22:754764.

3 Melby-Lervag M, Lyster SA, Hulme C: Phonological skills and their role in learning to read: a meta-analytic review. Psychol Bull 2012;138:322-352.

4 Peyrin C, Lallier M, Demonet JF, Pernet C, Baciu M, Le Bas JF, Valdois S: Neural dissociation of phonological and visual attention span disorders in developmental dyslexia: FMRI evidence from two case reports. Brain Lang 2012;120:381-394.

5 Beneventi H, Tonnessen FE, Ersland L, Hugdahl K: Executive working memory processes in dyslexia: behavioral and fMRI evidence. Scand J Psychol 2010;51:192-202.

6 Gathercole SE, Alloway TP, Willis C, Adams A: Working memory in children with reading disabilities. J Exp Child Psychol 2006;93:265281.

7 Menghini D, Carelsimo GA, Marotta L, Finzi A, Vicari S: Developmental dyslexia and explicit long-term memory. Dyslexia 2010;16: 213-225.

8 Menghini D, Finzi A, Carelsimo GA, Vicari S: Working memory impairment in children with developmental dyslexia: is it just a phonological deficity? Dev Neuropsychol 2011; 36:199-213

9 Smith-Spark JH, Fisk JE: Working memory functioning in developmental dyslexia. Memory $2007 ; 15: 34-56$.

10 Franceschini S, Gori S, Ruffino M, Pedrolli K, Facoetti A: A causal link between visual spatial attention and reading acquisition. Curr Biol 2012;22:814-819.

-11 Gabrieli JE, Norton E: Reading abilities: importance of visual-spatial attention. Curr Biol 2012;22:R298-R299.

12 Cohen-Mimran R, Sapir S: Auditory temporal processing deficits in children with reading disabilities. Dyslexia 2007;13:175-192.

13 Fitch RH, Szalkowski CE: Using animal models to dissociate genetic, neural, and behavioral contributors to language disability; in Benasich AA, Fitch RH (eds): Developmental Dyslexia: Early Precursors, Neurobehavioral Markers, and Biological Substrates. Baltimore, Brookes Publishing Company, 2012, pp 172-190.

14 Hamalainen JA, Salminen HK, Leppanen PH: Basic auditory processing deficits in dyslexia: systematic review of the behavioral and eventrelated potential/field evidence. J Learn Disabil DOI: 10.1177/0022219411436213.

15 Vandermosten M, Boets B, Luts H, Poelmans $\mathrm{H}$, Wouters J, Ghesquiere P: Impairments in speech and nonspeech sound categorization in children with dyslexia are driven by temporal processing difficulties. Res Dev Disabil 2011;32:593-603.

16 Galaburda AM, Sherman GF, Rosen GD, Aboitiz F, Geschwind N: Developmental dyslexia: four consecutive patients with cortical anomalies. Ann Neurol 1985;18:222-233.

17 Galaburda AM, Menard MT, Rosen GD: Evidence for aberrant auditory anatomy in developmental dyslexia. Proc Natl Acad Sci USA 1994;91:8010-8013.

18 Humphreys P, Kaufmann WE, Galaburda AM: Developmental dyslexia in women: neuropathological findings in three patients. Ann Neurol 1990;28:727-738.

19 Brambati SM, Termine C, Ruffino M, Stella G, Fazio F, Cappa SF, Perani D: Regional reductions of gray matter volume in familial dyslexia. Neurology 2004;63:742-745.

20 Kronbichler M, Wimmer H, Staffen W, Hutzler F, Mair A, Ladurner G: Developmental dyslexia: gray matter abnormalities in the occipitotemporal cortex. Hum Brain Mapp 2008;29:613-625.

21 Menghini D, Hagberg GE, Petrosini L, Bozzali M, Macaluso E, Caltagirone C, Vicari S: Structural correlates of implicit learning deficits in subjects with developmental dyslexia. Ann NY Acad Sci 2008;1145:212-221.

22 Siok WT, Niu Z, Jin Z, Perfetti CA, Tan LH: A structural-functional basis for dyslexia in the cortex of Chinese readers. Proc Natl Acad Sci USA 2008; 105:5561-5566.

23 Richlan F, Kronbichler M, Wimmer H: Structural abnormalities in the dyslexic brain: a meta-analysis of voxel-based morphometry studies. Hum Brain Mapp DOI: 10.1002/ hbm.22127.

24 Vandermosten M, Boets B, Wouters J, Ghesquiere P: A qualitative and quantitative review of diffusion tensor imaging studies in reading and dyslexia. Neurosci Biobehav Rev 2012;36:1532-1552.

25 Elnakib A, Casanova M, Gimelfarb G, Switala A, El-Baz A: Dyslexia diagnostics by 3D shape analysis of the corpus callosum. IEEE Trans Inf Technol Biomed 2012;16:700-708

26 Hasan KM, Molfese DL, Walimuni IS, Stuebing KK, Papanicolaou AC, Narayana PA, Fletcher JM: Diffusion tensor quantification and cognitive correlates of the macrostructure and microstructure of the corpus callosum in typically developing and dyslexic children. NMR Biomed 2012;25:1263-1270.

27 Paul LK: Developmental malformation of the corpus callosum: a review of typical callosal development and examples of developmental disorders with callosal involvement. J Neurodev Disord 2011;3:3-27.

28 Carter JC, Lanham DC, Cutting LE, Clements-Stephens AM, Chen X, Hadzipasic M, Kim J, Denckla MB, Kaufmann WE: A dual DTI approach to analyzing white matter in children with dyslexia. Psychiatry Res 2009; 172:215-219.
29 Hoeft F, McCandliss BD, Black JM, Gantman A, Zakerani N, Hulme C, Lyytinen H, Whitfield-Gabrieli S, Glover GH, Reiss AL, Gabrieli JD: Neural systems predicting long-term outcome in dyslexia. Proc Natl Acad Sci USA 2011;108:361-366.

-30 Pugh KR, Mencl WE, Jenner AR, Katz L, Frost SJ, Lee JR, Shaywitz SE, Shaywitz BA: Functional neuroimaging studies of reading and reading disability (developmental dyslexia). Ment Retard Dev Disabil Res Rev 2000;6:207213.

- 31 Rimrodt SL, Peterson DJ, Denckla MB, Kaufmann WE, Cutting LE: White matter microstructural differences linked to left perisylvian language network in children with dyslexia. Cortex 2010;46:739-749.

32 Wolf RC, Sambataro F, Lohr C, Steinbrink C, Martin C, Vasic N: Functional brain network abnormalities during verbal working memory performance in adolescents and young adults with dyslexia. Neuropsychologia 2010;48: 309-318.

33 Maisog JM, Einbinder ER, Flowers DL, Turkeltaub PE, Eden GF: A meta-analysis of functional neuroimaging studies of dyslexia. Ann NY Acad Sci 2008;1145:237-259.

34 Anthoni H, Sucheston LE, Lewis BA, TapiaPaez I, Fan X, Zucchelli M, Taipale M, Stein CM, Hokkanen ME, Castren E, Pennington BF, Smith SD, Olson RK, Tomblin JB, Schulte-Korne G, Nothen M, Schumacher J, Muller-Myhsok B, Hoffmann P, Gilger JW, Hynd GW, Nopola-Hemmi J, Leppanen PH, Lyytinen H, Schoumans J, Nordenskjold M, Spencer J, Stanic D, Boon WC, Simpson E, Makela S, Gustafsson JA, Peyrard-Janvid $\mathrm{M}$, Iyengar S, Kere J: The aromatase gene CYP19A1: several genetic and functional lines of evidence supporting a role in reading, speech and language. Behav Genet 2012; 42:509-527.

35 Francks C, Paracchini S, Smith SD, Richardson AJ, Scerri TS, Cardon LR, Marlow AJ, MacPhie IL, Walter J, Pennington BF, Fisher SE, Olson RK, DeFries JC, Stein JF, Monaco AP: A 77-kilobase region of chromosome 6 p22.2 is associated with dyslexia in families from the United Kingdom and from the United States. Am J Hum Genet 2004; 75:10461058.

36 Hannula-Jouppi K, Kaminen-Ahola N, Taipale M, Eklund R, Nopola-Hemmi J, Kaariainen $\mathrm{H}$, Kere J: The axon guidance receptor gene ROBO1 is a candidate gene for developmental dyslexia. PLoS Genet 2005; 1:e50.

- 37 Matsson H, Tammimies K, Zucchelli M, Anthoni H, Onkamo P, Nopola-Hemmi J, Lyytinen H, Leppanen PH, Neuhoff N, Warnke A, Schulte-Korne G, Schumacher J, Nothen MM, Kere J, Peyrard-Janvid M: SNP variations in the $7 \mathrm{q} 33$ region containing DGKI are associated with dyslexia in the Finnish and German populations. Behav Genet 2011;41: 134-140. 
-38 Meng H, Smith SD, Hager K, Held M, Liu J, Olson RK, Pennington BF, DeFries JC, Gelernter J, O’Reilly-Pol T, Somlo S, Skudlarski P, Shaywitz SE, Shaywitz BA, Marchione K, Wang Y, Paramasivam M, LoTurco JJ, Page GP, Gruen JR: DCDC2 is associated with reading disability and modulates neuronal development in the brain. Proc Natl Acad Sci USA 2005;102:17053-17058.

-39 Poelmans G, Engelen JJ, Van Lent-Albrechts J, Smeets HJ, Schoenmakers E, Franke B, Buitelaar JK, Wuisman-Frerker M, Erens W, Steyaert J, Schrander-Stumpel C: Identification of novel dyslexia candidate genes through the analysis of a chromosomal deletion. Am J Med Genet B Neuropsychiatr Genet 2009; 150B:140-147.

-40 Scerri TS, Paracchini S, Morris A, MacPhie IL, Talcott J, Stein J, Smith SD, Pennington BF, Olson RK, DeFries JC, Monaco AP, Richardson AJ: Identification of candidate genes for dyslexia susceptibility on chromosome 18 . PLoS One 2010;5:e13712.

-41 Taipale M, Kaminen N, Nopola-Hemmi J, Haltia T, Myllyluoma B, Lyytinen H, Muller K, Kaaranen M, Lindsberg PJ, Hannula-Jouppi K, Kere J: A candidate gene for developmental dyslexia encodes a nuclear tetratricopeptide repeat domain protein dynamically regulated in brain. Proc Natl Acad Sci USA 2003;100:11553-11558.

42 Paracchini S, Thomas A, Castro S, Lai C, Paramasivam M, Wang Y, Keating BJ, Taylor JM, Hacking DF, Scerri T, Francks C, Richardson AJ, Wade-Martins R, Stein JF, Knight JC, Copp AJ, Loturco J, Monaco AP: The chromosome $6 \mathrm{p} 22$ haplotype associated with dyslexia reduces the expression of KIAA0319, a novel gene involved in neuronal migration. Hum Mol Genet 2006;15:1659-1666.

-43 Rosen GD, Bai J, Wang Y, Fiondella CG, Threlkeld SW, LoTurco JJ, Galaburda AM: Disruption of neuronal migration by RNAi of Dyxlc1 results in neocortical and hippocampal malformations. Cereb Cortex 2007;17: 2562-2572.

44 Wang Y, Paramasivam M, Thomas A, Bai J, Kaminen-Ahola N, Kere J, Voskuil J, Rosen GD, Galaburda AM, Loturco JJ: DYX1C1 functions in neuronal migration in developing neocortex. Neuroscience 2006;143:515522.

-45 Dahdouh F, Anthoni H, Tapia-Paez I, Peyrard-Janvid M, Schulte-Korne G, Warnke A, Remschmidt H, Ziegler A, Kere J, MullerMyhsok B, Nothen MM, Schumacher J, Zucchelli M: Further evidence for DYX1C1 as a susceptibility factor for dyslexia. Psychiatr Genet 2009;19:59-63.

-46 Marino C, Citterio A, Giorda R, Facoetti A, Menozzi G, Vanzin L, Lorusso ML, Nobile M, Molteni M: Association of short-term memory with a variant within DYX1C1 in developmental dyslexia. Genes Brain Behav 2007;6: 640-646.
47 Smith SD, Pennington BF, Boada R, Shriberg LD: Linkage of speech sound disorder to reading disability loci. J Child Psychol Psychiatry 2005;46:1057-1066.

48 Wigg KG, Couto JM, Feng Y, Anderson B, Cate-Carter TD, Macciardi F, Tannock R, Lovett MW, Humphries TW, Barr CL: Support for EKN1 as the susceptibility locus for dyslexia on 15q21. Mol Psychiatry 2004;9: 1111-1121.

49 Marino C, Mascheretti S, Riva V, Cattaneo F, Rigoletto C, Rusconi M, Gruen JR, Giorda R, Lazazzera C, Molteni M: Pleiotropic effects of DCDC2 and DYX1C1 genes on language and mathematics traits in nuclear families of developmental dyslexia. Behav Genet 2011;41: 67-76.

50 Jamadar S, Powers NR, Meda SA, Gelernter J, Gruen JR, Pearlson GD: Genetic influences of cortical gray matter in language-related regions in healthy controls and schizophrenia. Schizophr Res 2011;129:141-148.

51 Threlkeld SW, McClure MM, Bai J, Wang Y, LoTurco JJ, Rosen GD, Fitch RH: Developmental disruptions and behavioral impairments in rats following in utero RNAi of Dyx1c1. Brain Res Bull 2007;71:508-514.

52 Szalkowski CE, Hinman JR, Threlkeld SW, Wang Y, LePack A, Rosen GD, Chrobak JJ, LoTurco JJ, Fitch RH: Persistent spatial working memory deficits in rats following in utero RNAi of Dyx1c1. Genes Brain Behav 2011;10: 244-252.

53 Fitch RH, Brown CP, Tallal P, Rosen GD: Effects of sex and MK-801 on auditory-processing deficits associated with developmental microgyric lesions in rats. Behav Neurosci 1997;111:404-412.

54 Peiffer AM, Rosen GD, Fitch RH: Sex differences in rapid auditory processing deficits in ectopic BXSB/MpJ mice. Neuroreport 2002; 13:2277-2280.

55 Peiffer AM, Rosen GD, Fitch RH: Sex differences in rapid auditory processing deficits in microgyric rats. Brain Res Dev Brain Res 2004;148:53-57.

56 Bai J, Ramos RL, Ackman JB, Thomas AM, Lee RV, LoTurco JJ: RNAi reveals doublecortin is required for radial migration in rat neocortex. Nat Neurosci 2003;6:1277-1283.

57 Chen F, LoTurco JJ: A method for stable transgenesis of radial glia lineage in rat neocortex by piggyBac mediated transposition. J Neurosci Methods 2012;207:172-180.

58 LoTurco JJ, Manent JB, Sidiqi F: New and improved tools for in utero electroporation studies of developing cerebral cortex. Cereb Cortex 2009;19:1120-i125.

-59 Fitch RH, Threlkeld SW, McClure MM, Peiffer AM: Use of a modified prepulse inhibition paradigm to assess complex auditory discrimination in rodents. Brain Res Bull 2008;76:1-7.
60 Faraday MM, Grunberg NE: The importance of acclimation in acoustic startle amplitude and pre-pulse inhibition testing of male and female rats. Pharmacol Biochem Behav 2000; 66:375-381.

61 Newbury AJ, Rosen GD: Genetic, morphometric, and behavioral factors linked to the midsagittal area of the corpus callosum. Front Genet 2012;3:91.

62 LoTurco J, Manent JB, Sidiqi F: New and improved tools for in utero electroporation studies of developing cerebral cortex. Cereb Cortex 2009;19 Suppl 1:i120-i125.

63 Jonasson Z: Meta-analysis of sex differences in rodent models of learning and memory: a review of behavioral and biological data. Neurosci Biobehav Rev 2005;28:811-825.

64 Boscariol M, Garcia VL, Guimaraes CA, Hage SR, Montenegro MA, Cendes F, Guerreiro MM: Auditory processing disorders in twins with perisylvian polymicrogyria. Arq Neuropsiquiatr 2009;67:499-501.

- 65 Boscariol M, Garcia VL, Guimaraes CA Montenegro MA, Hage SR, Cendes F, Guerreiro MM: Auditory processing disorder in perisylvian syndrome. Brain Dev 2010;32: 299-304.

66 Gaab N, Gabrieli JD, Deutsch GK, Tallal P, Temple E: Neural correlates of rapid auditory processing are disrupted in children with developmental dyslexia and ameliorated with training: an fMRI study. Restor Neurol Neurosci 2007;25:295310.

67 Witton C, Talcott JB, Hansen PC, Richardson AJ, Griffiths TD, Rees A, Stein JF, Green GG: Sensitivity to dynamic auditory and visual stimuli predicts nonword reading ability in both dyslexic and normal readers. Curr Biol 1998;8:791-797.

68 Benasich AA, Tallal P: Infant discrimination of rapid auditory cues predicts later language impairment. Behav Brain Res 2002;136:31-49.

69 Boets B, Vandermosten M, Poelmans H, Luts $\mathrm{H}$, Wouters J, Ghesquiere P: Preschool impairments in auditory processing and speech perception uniquely predict future reading problems. Res Dev Disabil 2011;32:560-570.

70 Fox AM, Reid CL, Anderson M, Richardson C, Bishop DV: Maturation of rapid auditory temporal processing and subsequent nonword repetition performance in children. Dev Sci 2012;15:204-211.

-71 Threlkeld SW, Hill CA, Rosen GD, Fitch RH: Early acoustic discrimination experience ameliorates auditory processing deficits in male rats with cortical developmental disruption. Int J Dev Neurosci 2009;27:321-328.

72 Hill CA, Threlkeld SW, Fitch RH: Early testosterone modulated sex differences in behavioral outcome following neonatal hypoxia ischemia in rats. Int J Dev Neurosci 2011;29: 381-388.

-73 Zhu C, Xu F, Wang X, Shibata M, Uchiyama Y, Blomgren K, Hagberg H: Different apoptotic mechanisms are activated in male and female brains after neonatal hypoxia-ischaemia. J Neurochem 2006;96:1016-1027.
Behavioral and Anatomical Changes in Male and Female Rats after RNAi for Dyx1c1
Dev Neurosci 2013;35:50-68 DOI: $10.1159 / 000348431$ 
74 Massinen S, Tammimies K, Tapia-Paez I, Matsson H, Hokkanen ME, Soderberg O, Landegren U, Castren E, Gustafsson JA, Treuter E, Kere J: Functional interaction of DYX1C1 with estrogen receptors suggests involvement of hormonal pathways in dyslexia. Hum Mol Genet 2009; 18:2802-2812.

75 Moores E, Cassim R, Talcott JB: Adults with dyslexia exhibit large effects of crowding, increased dependence on cues, and detrimental effects of distractors in visual search tasks. Neuropsychologia 2011;49:3881-3890.

76 Valdois S, Bidet-Ildei C, Lassus-Sangosse D, Reilhac C, N'guyen-Morel MA, Guinet E, Orliaguet JP: A visual processing but no phonological disorder in a child with mixed dyslexia. Cortex 2011;47:1197-1218.

77 Wright CM, Conlon EG, Dyck M: Visual search deficits are independent of magnocellular deficits in dyslexia. Ann Dyslexia 2012; 62:53-69.
8 Lobier M, Zoubrinetzky R, Valdois S: The visual attention span deficit in dyslexia is visual and not verbal. Cortex 2012;48:768-773.

79 Araujo AP: Attention deficit hyperactivity disorder and dyslexia: a history of overlap. Arq Neuropsiquiatr 2012;70:83-84.

80 Willcutt EG, Betjemann RS, McGrath LM, Chhabildas NA, Olson RK, DeFries JC, Pennington BF: Etiology and neuropsychology of comorbidity between $\mathrm{RD}$ and ADHD: the case for multiple-deficit models. Cortex 2010; 46:1345-1361.

81 Willcutt EG, Pennington BF, Duncan L, Smith SD, Keenan JM, Wadsworth S, Defries JC, Olson RK: Understanding the complex etiologies of developmental disorders: behavioral and molecular genetic approaches. J Dev Behav Pediatr 2010;31:533-544.

82 Currier TA, Etchegaray MA, Haight JL, Galaburda AM, Rosen GD: The effects of embryonic knockdown of the candidate dyslexia susceptibility gene homologue Dyx1c1 on the distribution of GABAergic neurons in the cerebral cortex. Neuroscience 2011;172:535546.
83 Peiffer AM, McClure MM, Threlkeld SW, Rosen GD, Fitch RH: Severity of focal microgyria and associated rapid auditory processing deficits. Neuroreport 2004;15:1923-1926.

84 Herman AE, Galaburda AM, Fitch RH, Carter AR, Rosen GD: Cerebral microgyria, thalamic cell size and auditory temporal processing in male and female rats. Cereb Cortex 1997;7:453-464.

85 Peiffer AM, Rosen GD, Fitch RH: Rapid auditory processing and MGN morphology in microgyric rats reared in varied acoustic environments. Dev Brain Res Brain Res 2002;138: 187-193.

86 Rosen GD, Mesples B, Hendriks M, Galaburda AM: Histometric changes and cell death in the thalamus after neonatal neocortical injury in the rat. Neuroscience 2006;141:875-888.

87 Galaburda AM, Livingstone M: Evidence for a magnocellular deficit in developmental dyslexia. Ann NY Acad Sci 1993;682:70-82. 